\title{
The Evens and Odds of CMB Anomalies
}

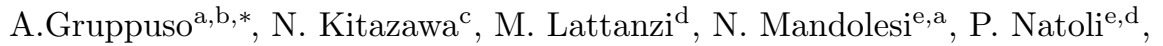 \\ A. Sagnottif \\ ${ }^{a}$ INAF-OAS Bologna, Osservatorio di Astrofisica e Scienza dello Spazio di Bologna, Istituto \\ Nazionale di Astrofisica, via Gobetti 101, I-40129 Bologna, Italy \\ ${ }^{b}$ INFN, Sezione di Bologna, Via Irnerio 46, I-40126 Bologna, Italy \\ ${ }^{c}$ Department of Physics, Tokyo Metropolitan University, Hachioji, Tokyo 192-0397, Japan \\ ${ }^{d}$ INFN - Sezione di Ferrara, Via Saragat 1, I-44100 Ferrara, Italy \\ ${ }^{e}$ Dipartimento di Fisica e Scienze della Terra, Università degli Studi di Ferrara, Via \\ Saragat 1, I-44100 Ferrara, Italy, INAF-IASF Bologna \\ ${ }^{f}$ Scuola Normale Superiore and INFN, Piazza dei Cavalieri 7 I-56126 Pisa, Italy
}

\begin{abstract}
The lack of power of large-angle CMB anisotropies is known to increase its statistical significance at higher Galactic latitudes, where a string-inspired preinflationary scale $\Delta$ can also be detected. Considering the Planck 2015 data, and relying largely on a Bayesian approach, we show that the effect is mostly driven by the even $-\ell$ harmonic multipoles with $\ell \lesssim 20$, which appear sizably suppressed in a way that is robust with respect to Galactic masking, along with the corresponding detections of $\Delta$. On the other hand, the first odd $-\ell$ multipoles are only suppressed at high Galactic latitudes. We investigate this behavior in different sky masks, constraining $\Delta$ through even and odd multipoles, and we elaborate on possible implications. We include low- $\ell$ polarization data which, despite being noise-limited, help in attaining confidence levels of about $3 \sigma$ in the detection of $\Delta$. We also show by direct forecasts that a future all-sky $E$ mode cosmic-variance-limited polarization survey may push the constraining power for $\Delta$ beyond $5 \sigma$.
\end{abstract}

Keywords: CMB, Low- $\ell$ anomaly, Inflation, Supergravity, String Theory

\footnotetext{
* Corresponding author

Email addresses: gruppuso@iasfbo.inaf.it (A.Gruppuso), noriaki.kitazawa@tmu.ac.jp (N. Kitazawa), lattanzi@fe.infn.it (M. Lattanzi), mandolesi@iasfbo.inaf.it (N. Mandolesi), paolo.natoli@unife.it (P. Natoli), sagnotti@sns.it (A. Sagnotti)
} 


\section{Introduction}

Cosmic Microwave Background (CMB) observations have been instrumental in defining the $\Lambda \mathrm{CDM}$ concordance model, and have constrained its parameters to the percent level or better [1. Anomalies in CMB temperature maps, however, have long surfaced, especially at large angular scales, with a typical 2 to $3 \sigma$ significance. Still, these anomalies are not a priori irrelevant in view of their potential physical implications. The lack of angular correlation in the CMB two-point function was originally noted in COBE data 2, and was later confirmed in all WMAP [3, 4, 5, 6, and Planck releases [7, 8, 9, The lowvariance anomaly $10,11,12$ is a related observation, suggesting that the low $-\ell$ CMB anisotropy contains less power, with respect to smaller angular scales, than $\Lambda \mathrm{CDM}$ would prefer. As pointed out by Copi et al. [13, if this behavior is not a statistical fluke only a physical mechanism impacting the CMB up to last scattering could explain it, while the integrated Sachs-Wolfe effect [14] would not be able to screen it.

In [15, 16] we have searched, in PLANCK temperature (and polarization) data, for signatures of modified primordial power spectra of the type

$$
P(k) \sim \frac{k^{3}}{\left[k^{2}+\Delta^{2}\right]^{2-\frac{n_{s}}{2}}} .
$$

Here $\Delta$ controls the transition from a large-scale depression to the usual ChibisovMukhanov tilt [17] $P(k) \sim k^{n_{s}-1}$. Starting from the slow-roll Coulomb-barrier Mukhanov-Sasaki potential [18, $\alpha / \eta^{2}$, where $\eta$ denotes conformal time and $\alpha=\left(n_{s}-3\right)\left(n_{s}-5\right) / 4$, the power spectrum of eq. (1) can be readily obtained via a vertical shift into $\alpha / \eta^{2}-\Delta^{2}$, which also models the sign change accompanying transitions from fast-roll to slow-roll. A power cut is indeed the main signature of an inflaton decelerating to slow-roll. It is also accompanied, in general, by a peak and some nearby oscillations, whose positions and sizes are however model dependent. 
String Theory [19] and Supergravity [20] may provide some clues on how slow-roll started. In orientifold models [21] with "brane supersymmetry breaking" 22, vacuum effects force a scalar to climb up a steep exponential potential as it emerges from the initial singularity, before attaining slow-roll in the ensuing descent [23]. Up to the local features that we have just mentioned, this dynamics gives rise to primordial power spectra exhibiting the cut of eq. (1), which could account for the observed low- $\ell$ depression of the CMB temperature angular power spectrum. This CMB anomaly might thus reflect an early epoch of deceleration, in principle accessible in scenarios with relatively few $e$-folds [24].

The string-inspired cosmology of 23$]^{1}$ (see [26] for reviews) motivated our work in [15, 16], where we showed that at higher Galactic latitudes the detection level of $\Delta$ in PLANCK data improves, reaching up a $99.4 \%$ C.L. in a blind $30^{\circ}$ extension of the standard mask (corresponding to an available sky fraction $f_{\text {sky }}=39 \%$ ), where one finds

$$
\Delta=(0.35 \pm 0.11) \times 10^{-3} \mathrm{Mpc}^{-1} \quad(68 \% \text { C.L. })
$$

The lack-of-power anomaly was known to grow when the Galactic mask is widened [12, 6, and $\Lambda$ CDM extended with eq. (1) nicely captures this feature. Sampling for $\Delta$ while altering the Galactic mask does not affect significantly the standard cosmological parameters, while $\Delta$ increases from $(0.17 \pm 0.09) \times$ $10^{-3} \mathrm{Mpc}^{-1}$ (68\% C.L.) to the result in eq. 22 in going from $f_{\text {sky }}=94 \%$ to $f_{\text {sky }}=39 \%$. Still, the dependence on the sky fraction of $\Delta-$ a parameter that is potentially of cosmological origin - appears intriguing. This very fact has led us to reconsider the issue from a different perspective, which is the purpose of the present work.

We thus continue our investigation of latitude effects, relying on the 2015 low - $\ell$ PLANCK likelihood [27, which was already used in [16] and is publicly

\footnotetext{
${ }^{1} \mathrm{~A}$ number of scenarios leading to infrared cuts were explored over the years, see 25 for a list of references.
} 
available from the Planck Legacy Archive 2. Our aim was initially a critical assessment of the current overall setup, in order to try and highlight local features that might or might not support further excisions near the Galactic plane, or perhaps local improvements of the component separation algorithms, for the sake of present and future observations. However, the scope of this inquiry has broadened somewhat along the way.

In detail, we focus on $\Delta$, both in view of its potential theoretical significance and, more phenomenologically, as a proxy for two well-known CMB anomalies, the lack of power and the even-odd asymmetry, and of their dependence on the sky fraction. To this end, we consider the same set of masks used in [16, which correspond to a sequence of blind extensions of the standard Commander [28, 29, 30. mask by six-degree steps 3 together with some additional "complementary" masks that we created, in combination with (anti)symmetrized temperature maps that disentangle the contributions of low- $\ell$ even and odd multipoles.

We believe that our main result, the different behaviour of even and odd multipoles, which was detected via different estimators, provides new insights into the low $-\ell$ tension between $\Lambda \mathrm{CDM}$ and the CMB. Moreover, we also confirm that this tension, which is largely driven by the even multipoles, increases at higher Galactic latitudes as described in [12, 6]. Initially we dwelled at length on a possible origin of the phenomenon from spurious contaminations around the Galactic plane, but the very behavior of even multipoles, which appear to conform to an isotropic pattern, strengthens somewhat the case for a primordial interpretation of $\Delta$.

In [16] we also attempted to place joint constraints on $\Delta$ and on a second parameter, $\gamma$, which was originally introduced in 24]. A positive value of

\footnotetext{
2 http://www.cosmos.esa.int/web/planck/pla

${ }^{3}$ Commander is one of four component separation algorithms used to analyze PLANCK data. Although we focus on Commander, which is employed in the standard PLANCK likelihood, for the sake of comparison, and in order to verify that our results are not unique to Commander, we have also analyzed a subset of cases with the Smica CMB temperature map [30], with consistent findings.
} 
$\gamma$ would model a peak located at the transition to an almost scale-invariant primordial power spectrum, albeit without nearby oscillations. We did find a scant evidence for a positive $\gamma$, but adding a second free parameter, as expected, weakened somewhat the detection level of $\Delta$. Here we are thus restricting our attention to $\Delta$, which suffices to model the large-scale depression that a decelerating inflaton would introduce in primordial power spectra.

The future of CMB experimental observations lies in providing high-quality cosmic-variance-limited polarization data. The main drive for this effort is at present the quest for $B$-modes. One of the purposes of this work is to stress that even high-quality $E$-mode polarization data, if fully exploited, can have far reaching implications. As we shall see, they can shed light on CMB anomalies, and thus potentially on the onset of inflation, via better determinations of the parameter $\Delta$ that is the main focus of this work.

The paper is organized as follows. In Section 2 we describe the adopted estimators, the tools used in the analysis, the datasets considered and the corresponding simulations. In Section 3 we describe our results for the cosmological parameters, paying special attention to $\Delta$ and to its connection with the evenodd asymmetry. We also report on our analysis for the three estimators of Section 2 and explore the constraining power of future all-sky polarization surveys. Finally, Section 4 collects some general considerations on this work and on its relation to future measurements, whose highlights are briefly summarized in Section 5 .

\section{Dataset and simulations}

Our analysis rests largely on the latest public Planck satellite CMB temperature data (2015 release) for $\ell<30$ and on a sequence of blind extensions of the standard PLANCK mask, together with some complements, whose features are summarized in table 1. It is worth stressing here that the PLANCK temperature measurements have already reached the cosmic variance limit, surely in the low-multipole range $\ell<30$ that is central to this work. 
Table 1: The masks that we have used, with the notation that identifies them. Here $f_{\text {sky }}$ denotes the sky fraction available for the analysis of the even+odd $T T$ contributions, while $f_{\text {sky }}^{ \pm}$denote the corresponding fractions available after parity (anti)symmetrization of the maps. The sky fractions of the last two columns refer to the same low- $\ell$ polarization mask, which relies on both PLANCK and WMAP9 data, as described in Section 2

\begin{tabular}{cccccc}
\hline Case & Label & $f_{\text {sky }}$ & $f_{\text {sky }}^{ \pm}$ & $f_{\text {sky }}^{\text {Pol }}$ & $f_{\text {sky }}^{\text {Pol }, \pm}$ \\
\hline$a$ & Standard & 93.6 & 90.7 & 73.9 & 65.3 \\
$b$ & Ext $_{06}$ & 83.8 & 83.0 & 73.9 & 65.3 \\
$c$ & Ext $_{12}$ & 70.8 & 70.6 & 73.9 & 65.3 \\
$d$ & Ext $_{18}$ & 59.1 & 59.1 & 73.9 & 65.3 \\
$e$ & Ext $_{24}$ & 48.7 & 48.7 & 73.9 & 65.3 \\
$f$ & Ext $_{30}$ & 39.4 & 39.4 & 73.9 & 65.3 \\
$g$ & $\operatorname{Ext}_{36}$ & 30.9 & 30.9 & 73.9 & 65.3 \\
$h$ & Compl $_{18}$ & 34.5 & 31.6 & 73.9 & 65.3 \\
$i$ & Compl $_{24}$ & 44.9 & 42.0 & 73.9 & 65.3 \\
$j$ & Compl $_{30}$ & 54.2 & 51.3 & 73.9 & 65.3 \\
$k$ & Compl $_{36}$ & 62.7 & 59.8 & 73.9 & 65.3 \\
\hline
\end{tabular}


The first row refers to the standard $f_{\text {sky }}=93.6 \%$ mask provided by PLANCK, rows $b-g$ refer to masks obtained via blind extensions of the standard one by $6^{\circ}$ steps, as in [16, and finally rows $h-k$ refer to masks obtained adjoining to the standard one in $a$ the complements of those in $d-g$. Notice that the (anti)symmetrization of maps reduces the sky fraction at the expense of regions close to the Galactic plane. Notice also that the polarization maps used were obtained combining WMAP9 and PLANCK data, as in 31, thus improving the signal-to-noise ratio while also extending the corresponding sky fractions. In the following, we shall refer to this combination as PLANCKLFI+WMAP, and to the corresponding mask as the "union" mask 4 .

In order to highlight the nature of these choices, fig. 1 displays the visible sky for the standard Commander mask in $a$, the visible sky for its blind extension in $f$ that resulted in the most significant determination of $\Delta$ in [16], and the complementary sky in $j$.

We have used the PLANCK temperature CMB map provided by the Commander algorithm, which enters the temperature sector of the low- $\ell$ PLANCK likelihood [27. This was smoothed at $440^{\prime}$ and was downsampled at HEALPix $]^{5}$ 32 resolution $N_{\text {side }}=16$. A Gaussian white noise realization with $\sigma=2 \mu \mathrm{K}$ was added to that map, spawning a noise covariance matrix $\mathcal{N}$ with elements $\mathcal{N}_{i j} \equiv \sigma^{2} \delta_{i j}$.

We proceeded along two different lines. We first performed Bayesian parameter estimation, both in the standard $\Lambda \mathrm{CDM}$ model and in its one-parameter extension, which we denote $\Lambda \mathrm{CDM} \Delta$. This also allowed to obtain Bayesian estimates of a few quantities that characterize large-scale anomalies:

- $S_{\frac{1}{2}}$, defined as 33

$$
S_{\frac{1}{2}}=\int_{\pi / 3}^{\pi} d \theta C(\theta)^{2} \sin \theta
$$

\footnotetext{
${ }^{4}$ We do not widen polarization masks, since current polarization data are already largely noise limited.

5 http://healpix.sourceforge.net/
} 
which is a standard tool to quantify the lack of correlation in the two-point function $C(\theta)$ for temperature CMB anisotropies, where

$$
C(\theta) \equiv \sum_{\ell=2}^{\ell_{\max }}\left(\frac{2 \ell+1}{4 \pi}\right) P_{\ell}(\cos \theta) C_{\ell}^{\mathrm{TT}} .
$$

Here $\ell_{\max }$ (=29 in our analysis) is the highest multipole considered, the $P_{\ell}$ are Legendre polynomials and the $C_{\ell}^{\mathrm{TT}}$ are temperature angular power spectrum coefficients. In practice, $S_{\frac{1}{2}}$ quantifies the distance between $C(\theta)$ and the null function, within the range $60^{\circ}<\theta<180^{\circ}$, and can be rewritten as 34.

$$
S_{\frac{1}{2}}=\sum_{\ell, \ell^{\prime}} C_{\ell}^{\mathrm{TT}} \mathcal{I}_{\ell \ell^{\prime}} C_{\ell^{\prime}}^{\mathrm{TT}}
$$

with

$$
\mathcal{I}_{\ell \ell^{\prime}}=\frac{(2 \ell+1)\left(2 \ell^{\prime}+1\right)}{(4 \pi)^{2}} \int_{-1}^{1 / 2} d x P_{\ell}(x) P_{\ell^{\prime}}(x) ;
$$

- the variance, used in 10, 11, 12,

$$
V\left(\ell_{\max }\right) \equiv C(0)=\sum_{\ell=2}^{\ell_{\max }}\left(\frac{2 \ell+1}{4 \pi}\right) C_{\ell}^{\mathrm{TT}}
$$

which defines the auto-correlation function up to $\ell_{\max }$;

- the even-odd asymmetry [35, 36, 37, 38, 7, defined as

$$
R\left(\ell_{\max }\right)=\frac{C^{+}\left(\ell_{\max }\right)}{C^{-}\left(\ell_{\max }\right)}
$$

where

$$
C^{ \pm}\left(\ell_{\max }\right)=\frac{1}{N_{ \pm}} \sum_{\ell=2}^{\ell_{\max }} \frac{1 \pm(-1)^{\ell}}{2} \frac{\ell(\ell+1)}{2 \pi} C_{\ell}^{\mathrm{TT}}
$$

and

$$
N_{+}=\left[\frac{\ell_{\max }}{2}\right], \quad N_{-}=\left[\frac{\ell_{\max }-1}{2}\right],
$$

with square brackets denoting integer parts. The quantity $R$ in eq. (8) compares the total amounts of power carried by even and odd multipoles, up to $\ell_{\max }$. 
In addition to the standard Commander temperature anisotropy map m, we also considered its point-parity even and odd projections $\mathbf{m}_{ \pm}$, which discretize

$$
\left(\frac{\delta T}{T}(\hat{n})\right)_{ \pm} \equiv \frac{1}{2}\left[\frac{\delta T}{T}(\hat{n}) \pm \frac{\delta T}{T}(-\hat{n})\right]
$$

where $\hat{n}$ identifies the direction corresponding to a given pixel. The resulting full-sky maps are displayed fig. 2

For given theoretical $C_{\ell}^{\mathrm{TT}}$, the likelihood function of the temperature map is, up to a normalization

$$
\mathcal{L}\left(\mathbf{m} \mid C_{\ell}^{\mathrm{TT}}\right) \propto \frac{1}{\left|\mathcal{C}^{\mathrm{TT}}\right|^{1 / 2}} \exp \left(-\frac{\mathbf{m}^{t}\left(\mathcal{C}^{\mathrm{TT}}\right)^{-1} \mathbf{m}}{2}\right) .
$$

Here $\mathcal{C}^{\mathrm{TT}}=\mathcal{S}^{\mathrm{TT}}+\mathcal{N}^{\mathrm{TT}}$ is the total temperature covariance matrix, including a signal matrix $\mathcal{S}^{\mathrm{TT}}$, with elements

$$
\mathcal{S}_{i j}^{\mathrm{TT}}=\sum_{\ell=2}^{4 N_{\text {side }}} \frac{2 \ell+1}{4 \pi} C_{\ell}^{\mathrm{TT}} P_{\ell}\left(\hat{n}_{i} \cdot \hat{n}_{j}\right)
$$

where $\hat{n}_{i}$ and $\hat{n}_{j}$ identify the directions pointing to pixels $i$ and $j$ and the $C_{\ell}$ for $\ell>29$ are fixed to fiducial values. Given that signal and noise in the original map are both Gaussian distributed, the even- and odd-parity maps are still Gaussian, and the corresponding likelihood functions are

$$
\mathcal{L}\left(\mathbf{m}_{ \pm} \mid C_{\ell}^{\mathrm{TT}}\right) \propto \frac{1}{\left|\mathcal{C}_{ \pm}^{\mathrm{TT}}\right|^{1 / 2}} \exp \left(-\frac{\mathbf{m}_{ \pm}^{t}\left(\mathcal{C}_{ \pm}^{\mathrm{TT}}\right)^{-1} \mathbf{m}_{ \pm}}{2}\right)
$$

where $\mathcal{C}_{ \pm}^{\mathrm{TT}} \equiv \mathcal{S}_{ \pm}^{\mathrm{TT}}+\mathcal{N}_{ \pm}^{\mathrm{TT}}$. Here

$$
\begin{aligned}
\mathcal{S}_{ \pm, i j}^{\mathrm{TT}} & =\sum_{\ell=2}^{4 N_{\text {side }}} \frac{2 \ell+1}{4 \pi} C_{\ell}^{\mathrm{TT}} P_{\ell}\left(\hat{n}_{i} \cdot \hat{n}_{j}\right) \frac{1 \pm(-1)^{\ell}}{2} \\
\mathcal{N}_{ \pm, i j}^{\mathrm{TT}} & =\frac{1}{2} \sigma^{2}\left[\delta_{i j} \pm \delta_{i p_{j}}\right]+\widetilde{\sigma}^{2} \delta_{i j}
\end{aligned}
$$

where the first result follows from the behavior under parity of the Legendre polynomials, and where $p_{j}$ identifies the index labeling the direction opposite to pixel $j$. The reader should notice the addition of a (small) diagonal second noise term: ${ }^{6}$ it regularizes the covariance matrix, which would be singular otherwise,

${ }^{6}$ We chose $\widetilde{\sigma}^{2}=0.01 \mu K^{2}$. 
since for technical reasons we found it convenient to work with the full datasets even in the presence of (anti)symmetrizations. Note also how eq. (15) implies that the temperature likelihood of the parity even (odd) map only depends on the even (odd) theoretical $C_{\ell}^{\mathrm{TT}}$ 's.

As explained in [39], taking into account the polarization brings along the other non-vanishing correlators $\langle T Q\rangle,\langle U U\rangle$ and $\langle Q Q\rangle$. This entails some complications, but it is important to explain how the even-odd separation works in this case, also in view of the forecasts of Section 3.4. The corresponding (anti)symmetrizations lead to

$$
\begin{aligned}
\left\langle T^{ \pm}(\hat{n}) Q^{ \pm}\left(\hat{n}^{\prime}\right)\right\rangle & =-\sum_{\ell} \frac{(2 \ell+1)}{4 \pi} \frac{1 \pm(-1)^{\ell}}{2} F_{\ell}^{10}(z) C_{\ell}^{\mathrm{TE}}, \\
\left\langle Q^{ \pm}(\hat{n}) Q^{ \pm}\left(\hat{n}^{\prime}\right)\right\rangle & =\sum_{\ell} \frac{(2 \ell+1)}{4 \pi}\left[\frac{1 \pm(-1)^{\ell}}{2} F_{\ell}^{12}(z) C_{\ell}^{\mathrm{EE}}\right. \\
& \left.-\frac{1 \mp(-1)^{\ell}}{2} F_{\ell}^{22}(z) C_{\ell}^{\mathrm{BB}}\right], \\
\left\langle U^{ \pm}(\hat{n}) U^{ \pm}\left(\hat{n}^{\prime}\right)\right\rangle & =\sum_{\ell} \frac{(2 \ell+1)}{4 \pi}\left[\frac{1 \mp(-1)^{\ell}}{2} F_{\ell}^{12}(z) C_{\ell}^{\mathrm{BB}}\right. \\
& \left.-\frac{1 \pm(-1)^{\ell}}{2} F_{\ell}^{22}(z) C_{\ell}^{\mathrm{EE}}\right],
\end{aligned}
$$

which enter the complete signal covariance matrix, and where

$$
\begin{aligned}
U^{ \pm}(\hat{n}) & =\frac{1}{2}[U(\hat{n}) \mp U(-\hat{n})], \\
Q^{ \pm}(\hat{n}) & =\frac{1}{2}[Q(\hat{n}) \pm Q(-\hat{n})],
\end{aligned}
$$

together with corresponding combinations for the noise. These results follow from the parity relations

$$
Q(\hat{n})+i U(\hat{n}) \longrightarrow Q(-\hat{n})-i U(-\hat{n})
$$

and from the symmetry properties

$$
\begin{aligned}
& F_{\ell}^{10}(-z)=(-1)^{\ell} F_{\ell}^{10}(z) \\
& F_{\ell}^{12}(-z)=(-1)^{\ell} F_{\ell}^{12}(z) \\
& F_{\ell}^{22}(-z)=(-1)^{\ell+1} F_{\ell}^{22}(z)
\end{aligned}
$$


with $z=\hat{n} \cdot \hat{n}^{\prime}$, which are listed in the Appendix of [39]. Notice that the correlation functions in eq. (17) are adapted to frames aligned to the maximal circles joining the points identified by $\hat{n}$ and $\hat{n}^{\prime}$. The actual results in standard frames with one axis aligned with the Galactic plane can then be obtained making use of the rotation matrices in [39].

We derived parameter estimates using the Monte Carlo engine CosmoMC [40], interfaced with a modified version of the Boltzmann code camb [41, and with a pixel-based low- $\ell(\ell<30)$ likelihood code implementing eqs. 12$)$ and 114$)$ or, more precisely, their versions extended to include polarization. We also included information from the high- $\ell(\ell \geq 30)$ temperature power spectrum and from the lensing potential from Planck, processed through the publicly available likelihood released by the PLANCK collaboration [27]. Notice that, since the preceding analysis of [15, 16] resulted in the determination of $\Delta$ in eq. (2), which only impacts very large scales, we refrain from (anti)symmetrizing the high $-\ell$ likelihood in this analysis.

We also performed a complementary frequentist analysis, in order to highlight the tension between data and the predictions of $\Lambda \mathrm{CDM}$. To this end, we simulated 10000 CMB-plus-noise maps, extracting their signal contributions from the PLANCK fiducial $\Lambda \mathrm{CDM}$ angular power spectrum. We analyzed these maps and the observed Commander map with an optimal angular power spectrum estimator, BolPol [42, for the whole sequence of masks. With the resulting

spectra, we built the estimators of eqs. (3) and (7), and the joint probability $P\left[C^{+}\left(\ell_{\max }\right), C^{-}\left(\ell_{\max }\right)\right]$, where the $C^{ \pm}$are defined in eq. (9), automatically accounting for cosmic variance and for the increase of sampling variance that accompanies reduced sky fractions.

\section{Results}

We can now turn to a description of our results. We first present the constraints on the cutoff scale $\Delta$ and the standard $\Lambda$ CDM parameters and then turn to the three estimators of Section 2 . We conclude our discussion with a frequen- 
tist assessment of the tension between low $-\ell$ data and $\Lambda \mathrm{CDM}$, paying special attention to disentangling the contributions of even and odd multipoles, and with some forecasts for future experiments aimed at high-quality polarization data.

\subsection{Constraints on $\Delta$}

In this section we provide the posterior distributions for the scale $\Delta$ of eq. (1) and the six $\Lambda \mathrm{CDM}$ parameters, namely the angle $\theta_{s}$ subtended by the sound horizon at recombination, the baryon density $\Omega_{b} h^{2}$, the cold dark matter density $\Omega_{c} h^{2}$, the logarithmic amplitude $\log \left(10^{10} A_{s}\right)$, the spectral index $n_{s}$ and the reionization optical depth $\tau$.

As explained in Section 2, for all masks listed in table 1, we derived our constraints also considering the low- $\ell$ likelihood $(\ell \leq 29)$ based on the full (even plus odd) spectrum. In detail, we resorted to the extensions of eqs. 12 and $(13)$ that include polarization and to their counterparts corresponding to eqs. (14), (15) and (16), which only involve even or odd multipoles. Our results are collected in table 2 , where we denote with $\Delta$ the estimates derived from the "even + odd" dataset and with $\Delta_{+}\left(\Delta_{-}\right)$those derived from its "even" ("odd") subsets. We shall stick to this notation also in the following.

Fig. 3 collects the estimates for the parameter $\Delta$ obtained considering the standard and extended masks of cases $a-g$ in table 1. We use blue circles when we employ $\mathbf{m}$ ("even + odd" multipoles), orange squares when we employ $\mathbf{m}_{+}$ ("even" multipoles), and finally green triangles when we employ $\mathbf{m}_{-}$("odd" multipoles).

Notice that:

- $\Delta$ shifts toward higher values at higher Galactic latitudes, attaining a maximum statistical significance in the $\mathrm{Ext}_{30}$ mask, as already found in our previous work [16;

- the mean value of $\Delta_{+}$is always larger than the mean value of $\Delta_{-}$, with only one exception, in the $\operatorname{Ext}_{36}$ mask. Moreover, $\Delta_{+}$is statistically larger 
Table 2: Constraints on the scale $\Delta$ for the various masks in table 1 that were used in our analysis. $\Delta, \Delta_{+}$and $\Delta_{-}$refer to estimates from the "even+odd", "even", and "odd" datasets. When the estimate of $\Delta$ differs from zero at more than $95 \%$ confidence level, we report it in the form of mean $\pm 68 \%$ uncertainty; otherwise, we only report the $95 \%$ upper limit.

\begin{tabular}{ccccc}
\hline Case & Label & $10^{3} \Delta\left[\mathrm{Mpc}^{-1}\right]$ & $10^{3} \Delta_{+}\left[\mathrm{Mpc}^{-1}\right]$ & $10^{3} \Delta_{-}\left[\mathrm{Mpc}^{-1}\right]$ \\
\hline$a$ & Standard & $<0.31$ & $0.31_{-0.11}^{+0.13}$ & $<0.26$ \\
$b$ & $\operatorname{Ext}_{06}$ & $<0.32$ & $0.32_{-0.12}^{+0.13}$ & $<0.26$ \\
$c$ & Ext $_{12}$ & $0.22_{-0.09}^{+0.10}$ & $0.36_{-0.12}^{+0.13}$ & $<0.30$ \\
$d$ & Ext $_{18}$ & $0.28_{-0.09}^{+0.11}$ & $0.38 \pm 0.13$ & $<0.33$ \\
$e$ & $\operatorname{Ext}_{24}$ & $0.33 \pm 0.10$ & $0.39_{-0.14}^{+0.15}$ & $<0.41$ \\
$f$ & $\operatorname{Ext}_{30}$ & $0.36_{-0.10}^{+0.11}$ & $0.36_{-0.14}^{+0.16}$ & $<0.52$ \\
$g$ & $\operatorname{Ext}_{36}$ & $0.33 \pm 0.13$ & $0.32 \pm 0.15$ & $0.34_{-0.15}^{+0.17}$ \\
$h$ & $\mathrm{Compl}_{18}$ & $<0.29$ & $<0.48$ & $<0.22$ \\
$i$ & $\mathrm{Compl}_{24}$ & $<0.29$ & $<0.46$ & $<0.22$ \\
$j$ & $\mathrm{Compl}_{30}$ & $<0.30$ & $0.27_{-0.12}^{+0.13}$ & $<0.24$ \\
$k$ & $\mathrm{Compl}_{36}$ & $<0.28$ & $0.28_{-0.12}^{+0.13}$ & $<0.23$ \\
\hline
\end{tabular}


than zero at more than $2 \sigma$ in all masks, while $\Delta_{-}$is always statistically compatible with zero within $2 \sigma$, with one exception in the Ext $_{36}$ mask. All in all, the even multipoles drive the detection of $\Delta$ in [16] in a way that is largely independent of the mask;

- more in detail, $\Delta_{-}$is statistically compatible with zero within $1 \sigma$ in all masks from $a$ to $d$, and then increases monotonically. On the other hand, $\Delta_{+}$increases slightly up to the $e$ mask, to then decrease slightly in the $f$ and $g$ masks.

Fig. 4 compares the estimates for $\Delta$ obtained in the complementary masks $h-k$ of table 1 The reader should appreciate the striking stability of these results, which conforms to the pattern that already emerged: even multipoles contain typically less power than odd ones.

$\Delta_{+}$and $\Delta_{-}$can be used to trace the even-odd asymmetry, which we characterize via

$$
\frac{\Delta_{+}-\Delta_{-}}{\sqrt{\sigma_{+}^{2}+\sigma_{-}^{2}}},
$$

with $\sigma_{+}\left(\sigma_{-}\right)$the standard deviation of $\Delta_{+}\left(\Delta_{-}\right)$. This quantity, shown in fig. 5, is close to the $2 \sigma$ level up to the $\operatorname{Ext}_{24}$ mask, and then decreases below the $1 \sigma$ level in the $\operatorname{Ext}_{30}$ and Ext 36 masks.

Figs. 6 and 7 illustrate the behaviour of the six $\Lambda \mathrm{CDM}$ parameters for three significant mask choices ( $a$ and $f$ of table 1). Each figure contains 6 posteriors for each parameter with the usual color code: blue, orange and green refer to even+odd, even and odd contributions for the low- $\ell$ likelihood. Moreover, dashed lines refer to $\Lambda \mathrm{CDM}$, while solid ones refer to $\Lambda \mathrm{CDM} \Delta$. In general, the introduction of $\Delta$, which captures the lack of power, tends to improve the stability of the other parameters. The continuous lines, which reflect "even+odd", "even" and "odd" contributions, are indeed largely superposed in all cases. On the other hand, the green dashed lines, which reflect odd multipoles in $\Lambda$ CDM, are very close to the solid ones in figs. 6 and move only slightly in fig. 7. Finally, $\Delta$ brings along slight shifts of $n_{s}$ and $A_{s}$, consistently with its role in accounting 
for the low- $\ell$ lack of power.

As shown in [15, 16], the introduction of the parameter $\Delta$ impacts only the lowest multipoles. In order to highlight this feature, in fig. 8 we have displayed $2 \mathrm{D}$ contour plots of $\Delta v s$ the band powers $D_{\ell}=\frac{\ell(\ell+1)}{2 \pi} C_{\ell}$, for $\ell=$ $2,5,8,11,14,17$ and 20 . There is clearly a high degree of anti-correlation between $\Delta$ and $D_{\ell}$ for the lowest multipoles, which fades out for higher values of $\ell$. The separation between the two regimes occurs for $\ell \sim 14$ in the standard mask, and for $\ell \sim 18$ in the Ext 30 mask. Beyond these thresholds, the values of $D_{\ell}$ become very weakly sensitive to $\Delta$. These results are nicely compatible with what found in fig. 5 of [16.

Similarly, figs. 9 and 10 show the 2D contour plots of $\Delta$ vs $D_{\ell}$ obtained taking into account only the "even" or "odd" parts of the likelihood function. Notice that the level of anti-correlation of these cases is similar to what already found in fig. 8. Moreover, consistently with our previous findings, one can see again that the "even" part pushes $\Delta$ towards larger values, making it more significantly different from zero. On the other hand, the "odd" part tends to make $\Delta$ more compatible with zero, although the significance of its detection increases somewhat in wider masks.

The upper panel in fig. 11 displays the fiducial models obtained with complete $\ell<30$ datasets corresponding to the Standard mask, to the $\operatorname{Ext}_{24}$ mask and to the Ext $_{30}$ mask. The middle panel compares the fiducial models obtained for the $\mathrm{Ext}_{24}$ mask using even, odd and even+odd datasets for $\ell<30$. Finally, the lower panel compares the fiducial models obtained for the Ext $_{30}$ mask using even, odd and even+odd datasets for $\ell<30$.

Tables 3 and 4 contain the results of the Akaike tests, obtained computing

$$
\mathrm{AIC}=2 k-2 \log \mathcal{L}_{\max },
$$

where $k$ is the number of parameters used in the fit to obtain the maximum likelihood $\mathcal{L}_{\text {max }}$, and the corresponding probabilities $P$ for the two models at stake, $\Lambda \mathrm{CDM}$ and $\Lambda \mathrm{CDM} \Delta$. Consistently with our other results here and in [15, 16, notice the preference, within $\Lambda \operatorname{CDM} \Delta$, for the $\operatorname{Ext}_{30}$ mask in the full 
Table 3: Akaike tests for the extended masks in table 1 and corresponding probabilities $P$ for the two models that we are considering $(\Lambda \mathrm{CDM}$ and $\Lambda \mathrm{CDM} \Delta)$. Here $\delta \mathrm{AIC}=$ $\mathrm{AIC}_{\Lambda \mathrm{CDM}}-\mathrm{AIC}_{\Lambda \mathrm{CDM} \Delta}$.

\begin{tabular}{|c|c|c|c|c|c|}
\hline Case & Label & dataset & $\delta \mathrm{AIC}$ & $P(\Lambda \mathrm{CDM})$ & $P(\Lambda \mathrm{CDM} \Delta)$ \\
\hline$a$ & Standard & full & -0.680 & 0.584 & 0.416 \\
\hline$a$ & Standard & even & 1.908 & 0.278 & 0.722 \\
\hline$a$ & Standard & odd & -0.630 & 0.578 & 0.422 \\
\hline$b$ & $\operatorname{Ext}_{06}$ & full & -1.172 & 0.642 & 0.358 \\
\hline$b$ & $\mathrm{Ext}_{06}$ & even & 3.848 & 0.127 & 0.873 \\
\hline$b$ & $\operatorname{Ext}_{06}$ & odd & -2.522 & 0.779 & 0.221 \\
\hline$c$ & Ext $_{12}$ & full & 0.540 & 0.433 & 0.567 \\
\hline$c$ & $\operatorname{Ext}_{12}$ & even & 3.752 & 0.133 & 0.867 \\
\hline$c$ & $\mathrm{Ext}_{12}$ & odd & -2.802 & 0.802 & 0.198 \\
\hline$d$ & $\operatorname{Ext}_{18}$ & full & 3.020 & 0.181 & 0.819 \\
\hline$d$ & $\mathrm{Ext}_{18}$ & even & 4.289 & 0.105 & 0.895 \\
\hline$d$ & $\operatorname{Ext}_{18}$ & odd & -2.373 & 0.766 & 0.234 \\
\hline$e$ & $\operatorname{Ext}_{24}$ & full & 2.418 & 0.230 & 0.770 \\
\hline$e$ & $\mathrm{Ext}_{24}$ & even & 3.359 & 0.157 & 0.843 \\
\hline$e$ & Ext $_{24}$ & odd & -2.199 & 0.750 & 0.250 \\
\hline$f$ & $\mathrm{Ext}_{30}$ & full & 4.750 & 0.085 & 0.915 \\
\hline$f$ & Ext $_{30}$ & even & 1.205 & 0.354 & 0.646 \\
\hline$f$ & $\mathrm{Ext}_{30}$ & odd & -0.657 & 0.581 & 0.419 \\
\hline$g$ & Ext $_{36}$ & full & 2.156 & 0.254 & 0.746 \\
\hline$g$ & $\operatorname{Ext}_{36}$ & even & 1.103 & 0.366 & 0.634 \\
\hline$g$ & $\operatorname{Ext}_{36}$ & odd & -0.286 & 0.536 & 0.464 \\
\hline
\end{tabular}


Table 4: Akaike tests for the complementary masks in table 1 and corresponding probabilities $P$ for the two models that we are considering ( $\Lambda$ CDM and $\Lambda$ CDM $\Delta$ ). Here $\delta \mathrm{AIC}=\mathrm{AIC}_{\Lambda \mathrm{CDM}}-\mathrm{AIC}_{\Lambda \mathrm{CDM} \Delta}$.

\begin{tabular}{cccrcc}
\hline Case & Label & dataset & $\delta$ AIC & $P(\Lambda C D M)$ & $P(\Lambda \mathrm{CDM} \Delta)$ \\
\hline$h$ & $\mathrm{Compl}_{18}$ & full & -1.408 & 0.669 & 0.331 \\
$h$ & $\mathrm{Compl}_{18}$ & even & 0.725 & 0.410 & 0.590 \\
$h$ & $\mathrm{Compl}_{18}$ & odd & -1.376 & 0.666 & 0.334 \\
$i$ & $\mathrm{Compl}_{24}$ & full & -0.294 & 0.537 & 0.463 \\
$i$ & $\mathrm{Compl}_{24}$ & even & -0.433 & 0.554 & 0.446 \\
$i$ & $\mathrm{Compl}_{24}$ & odd & -3.203 & 0.832 & 0.168 \\
$j$ & $\mathrm{Compl}_{30}$ & full & -0.290 & 0.536 & 0.464 \\
$j$ & $\mathrm{Compl}_{30}$ & even & 0.540 & 0.433 & 0.567 \\
$j$ & $\mathrm{Compl}_{30}$ & odd & -2.930 & 0.812 & 0.188 \\
$k$ & $\mathrm{Compl}_{36}$ & full & -2.436 & 0.772 & 0.228 \\
$k$ & $\mathrm{Compl}_{36}$ & even & 2.462 & 0.226 & 0.774 \\
$k$ & $\mathrm{Compl}_{36}$ & odd & -2.497 & 0.777 & 0.223 \\
\hline
\end{tabular}


case and for the Ext 18 mask in the even case. When only odd multipoles are considered, $\Lambda \mathrm{CDM}$ is preferred in almost all cases.

Tables 5 and 6 collect the detection levels of $\Delta$ for all the extended and complementary masks that we considered, whose properties are summarized in table 1. Fig. 12 displays the results in the last column of table 5

One may wonder about the relevance of accounting for low $-\ell$ polarization, especially in view of its low signal-to-noise level in both WMAP and PLANCK data. In order to address this question, we repeated our analysis relying only on temperature data and assuming a Gaussian prior for $\tau=0.070 \pm 0.015$. Notice, in fact, that $\tau, A_{s}$ and $\Delta$ all impact on large-scale power. In particular, the posteriors for $A_{s}$ and $\tau$ tend to shift when $\Delta$ is sampled, in ways that are more sensitive to temperature for the former and to polarization for the latter. It is thus preferable to use low- $\ell$ polarization and let the data adjust parameters accordingly. In the presence of a $\tau$ prior, instead, the data are bound to accept it, and consequently both $\Delta$ and $A_{s}$ shift a bit. The $\left(A_{s}, \tau, \Delta\right)$ correlation pattern is manifest in the three panels of fig. 13. With a $\tau$ prior the detection level of $\Delta$ in the Ext $_{24}$ mask would become slightly higher than in Ext $_{30}$ mask, which was instead favoured by the complete analysis in [16, and is favoured again here, where we resort to the joint PLANCKLFI+WMAP dataset in polarization, following [31]. A similar argument would apply if one left out the lensing likelihood, which impacts on $A_{s}$, and hence on $\tau$ and $\Delta$. For this reason, we preferred to feed both low- $\ell$ polarization and lensing information in our analysis.

At any rate, had we only relied on priors, our results would have been affected only slightly, and our conclusions would have been essentially the same 7

\footnotetext{
${ }^{7}$ The PLANCK collaboration published 43 the estimate $\tau=0.055 \pm 0.009$, which is significantly smaller than what the PLANCK (and WMAP9) publicly available polarization likelihood, which we use, would imply. In order to check that this lower value of $\tau$ does not affect our conclusions, we also performed an analysis using only the temperature portion of the likelihood of eqs. 12 and 13 , along with a $\tau$ prior derived from the above estimate. This choice has again no appreciable effects on our conclusions.
} 
Table 5: Detection levels of $\Delta$ for the extended masks of Table 1, There are a few differences with respect to [16] since here we rely, for the low- $\ell$ polarization, on the joint PlanckLFI+WMAP dataset described in [31. Some determinations improved slightly, along the lines of what we shall discuss in Section 3.4 due to the larger sky fraction thus available in polarization.

\begin{tabular}{ccccc}
\hline Case & Label & dataset & Detection Level $(\%)$ & Detection Level $(\sigma)$ \\
\hline \hline$a$ & Standard & full & 93.26 & 1.83 \\
$a$ & Standard & even & 98.59 & 2.46 \\
$a$ & Standard & odd & 52.52 & 0.72 \\
$b$ & Ext $_{06}$ & full & 92.30 & 1.77 \\
$b$ & Ext $_{06}$ & even & 98.65 & 2.47 \\
$b$ & Ext $_{06}$ & odd & 41.03 & 0.54 \\
$c$ & Ext $_{12}$ & full & 96.41 & 2.10 \\
$c$ & Ext $_{12}$ & even & 99.39 & 2.74 \\
$c$ & Ext $_{12}$ & odd & 18.93 & 0.24 \\
$d$ & Ext $_{18}$ & full & 99.15 & 2.63 \\
$d$ & Ext $_{18}$ & even & 99.23 & 2.67 \\
$d$ & Ext $_{18}$ & odd & 69.80 & 1.03 \\
$e$ & Ext $_{24}$ & full & 99.32 & 2.71 \\
$e$ & Ext $_{24}$ & even & 99.05 & 2.59 \\
$e$ & Ext $_{24}$ & odd & 81.57 & 1.33 \\
$f$ & Ext $_{30}$ & full & 99.84 & 3.16 \\
$f$ & Ext $_{30}$ & even & 98.47 & 2.43 \\
$f$ & Ext $_{30}$ & odd & 94.37 & 1.91 \\
$g$ & Ext $_{36}$ & full & 98.60 & 2.46 \\
$g$ & Ext $_{36}$ & even & 96.27 & 2.08 \\
$g$ & Ext $_{36}$ & odd & 96.60 & \\
\hline & & & & 2.12 \\
\hline
\end{tabular}


Table 6: Detection levels of $\Delta$ for the complementary masks of Table 1

\begin{tabular}{ccccc}
\hline Case & Label & dataset & Detection Level $(\%)$ & Detection Level $(\sigma)$ \\
\hline \hline$h$ & $\mathrm{Compl}_{18}$ & full & 80.29 & 1.29 \\
$h$ & $\mathrm{Compl}_{18}$ & even & 94.70 & 1.94 \\
$h$ & $\mathrm{Compl}_{18}$ & odd & 9.36 & 0.12 \\
$i$ & $\mathrm{Compl}_{24}$ & full & 78.54 & 1.24 \\
$i$ & $\mathrm{Compl}_{24}$ & even & 94.43 & 1.91 \\
$i$ & $\mathrm{Compl}_{24}$ & odd & 24.70 & 0.32 \\
$j$ & $\mathrm{Compl}_{30}$ & full & 86.96 & 1.51 \\
$j$ & $\mathrm{Compl}_{30}$ & even & 96.28 & 2.08 \\
$j$ & $\mathrm{Compl}_{30}$ & odd & 0.00 & 0 \\
$k$ & $\mathrm{Compl}_{36}$ & full & 82.46 & 1.36 \\
$k$ & $\mathrm{Compl}_{36}$ & even & 97.41 & 2.23 \\
$k$ & $\mathrm{Compl}_{36}$ & odd & 0.00 & 0 \\
\hline
\end{tabular}

It is however remarkable how low- $\ell$ polarization has noticeable effects on the detection of $\Delta$, even with the present-day low signal-to-noise ratio. The constraining power of the higher-quality polarization data awaited from the next generation of experiments [44, 45, 46, in general and on $\Delta$ in particular, remains to be ascertained, but it is potentially appealing, as we shall see shortly.

\subsection{Bayesian constraints on $S_{\frac{1}{2}}, V$ and $R$}

For each mask in table 1 we have built the posterior distribution functions of the estimators $S_{\frac{1}{2}}, V$ and $R$ defined in Section 2 considering "even+odd", "even" and "odd" contributions.

The posteriors of $S_{\frac{1}{2}}$ are collected in fig. 14 , where the first column refers to $\Lambda \mathrm{CDM}$ while the second refers to $\Lambda \mathrm{CDM} \Delta$. In both cases, the upper panels are for "even+odd", the middle ones are for "even" and the lower ones are for "odd". While the posteriors in the first column are quite stably centered at about $38000 \mu \mathrm{K}^{4}$, the others display a richer behaviour, with a second peak around 
$6000 \mu \mathrm{K}^{4}$ that becomes readily dominant for wider masks. Consistently with our preceding findings, the "odd" part is more resilient to converging toward the lower peak. The same pattern presents itself for the other two estimators $V$ and $R$, and the corresponding plots are collected in figs. 15 and 16 .

\subsection{Comments on the frequentist approach}

In principle, one can also build the preceding estimators via a frequentist approach, in order to test the $\Lambda \mathrm{CDM}$ model. In this case, however, wider masks and the corresponding increased sampling variance bring along negative values for some $C_{\ell}^{\mathrm{TT}}$ 's. This is particularly unpleasant for the ratio $R$ of eq. 8 , and all in all this approach is less suitable for our purposes. Consequently, as an example we show in fig. 17 the $2 \mathrm{D}$ contour plot of $C^{+} v s C^{-}$for the mask $\mathrm{Ext}_{18}$. The black dot identifies the expected values for $C^{+}$and $C^{-}$in a fiducial $\Lambda \mathrm{CDM}$ model, while the red one identifies the observed values. Moreover, the contours identify the levels at 1,2 and $3 \sigma$ for the empirical distribution expected in $\Lambda \mathrm{CDM}$. The resulting information is along the lines of what we already said: the "even" contribution lies well below $\Lambda \mathrm{CDM}$, while the "odd" one is largely compatible with it.

\subsection{Forecasts for future experiments}

Several projects for all-sky future experiments are currently under development, as the JAXA-led LiteBIRD [44, or are being proposed, as the European CORE [47. Their observations should provide cosmic-variance-limited measurements of the CMB $E$-mode polarization field, at least in the low- $\ell$ region. In order to explore the prospects that they offer for better determinations of $\Delta$, or for ruling it out altogether, we model their expected polarization maps adding only a diagonal regularization noise contribution of $0.01 \mu \mathrm{K}^{2}$ in both $Q$ and $U$ to a pixel windowed, $N_{\text {side }}=16 \mathrm{CMB}$ field. The latter is a realization of a fiducial cosmological mode 8 with $\Delta=0.37 \times 10^{-3} \mathrm{Mpc}^{-1}$. This treatment assumes ideal component separation and negligible systematic effects at the level

\footnotetext{
${ }^{8}$ In particular, we take as our fiducial the best-fit model obtained in the Ext $_{30}$ mask.
} 
of $E$-modes, a goal that lies within reach for the next generation space-borne experimental efforts [44, 47, 48, 49].

Our results are presented in fig. 18, where we collect some forecasts for the detection levels of $\Delta$ within the setup just discussed. All results are obtained from simulated low $-\ell$ datasets that share the same underlying CMB realization, but are associated to different noise levels and different maskings. These simulated low- $\ell$ datasets are combined with the actual high- $\ell$ and lensing PLANCK data. To begin with, the gray curve refers to a dataset with the same noise properties as real data, but using the standard and union masks in temperature and polarization, which could result in a detection level of $\Delta$ of about $3.5 \sigma$. On the other hand an ideal experiment with the same masking, but measuring the large-scale polarization down to the cosmic variance limit could raise the detection level for $\Delta$ up to about $6 \sigma$ (orange curve). Finally, the same cosmic-variance limited data, analyzed through the standard PLANCK mask in temperature and with no masking in polarization could result in detection levels even slightly beyond $6 \sigma$ (blue curve).

\section{Final remarks}

We have presented new evidence that some statistical properties of the CMB anisotropy depend sizeably on the Galactic latitude. To begin with, we have confirmed that at higher latitudes the observed lack of power is more pronounced than in standard masking. We have characterised this feature via the primordial power spectra

$$
P(k) \sim \frac{k^{3}}{\left[k^{2}+\Delta^{2}\right]^{2-\frac{n_{s}}{2}}},
$$

which approach $P(k) \sim k^{n_{s}-1}$ for large values of $k$, and where the scale $\Delta$ accounts for the low- $\ell$ lack of power. Its determination translates, as in [16], in departures from $\Lambda \mathrm{CDM}$ that depend on Galactic masking, and become sizeable below $\ell \sim 7$ for the standard mask and below $\ell \sim 15$ for the extended mask Ext 30 of table 1. Moreover, we have discovered that the latitude dependence is largely due to the odd multipoles, which contain more power around the Galactic plane 
than away from it. On the other hand, the even multipoles appear relatively stable in this respect, and lead to a determination of $\Delta$ that is essentially latitude independent, consistently with its possible cosmological origin. This behavior translates into an even-odd asymmetry that is largely concentrated around the Galactic plane.

One cannot exclude that low $-\ell$ anomalies be a statistical fluke at the $3-\sigma$ level. The even-odd differences that we are highlighting could also be induced by unidentified systematic contaminations at very large scales that fade out as one moves away from the Galactic plane. However, it seems difficult to envisage systematic effects that could impart an even-odd asymmetry on the CMB sky. For instance, standard diffuse Galactic emissions are typically symmetric with respect to the Galactic plane, and ought to enhance even multipoles with respect to odd ones (see eq. (44), a behaviour that is opposite to what we are detecting. In other words, if this signature were due to unaccounted foregrounds, these ought to be distributed antisymmetrically, in particular with respect to antipodal points around the Galactic plane. Residuals of unaccounted asymmetric foregrounds, however, are not the only candidates for such an even-odd pattern, which might also originate from systematic effects of instrumental origin, or from a tricky combination of both. The fact that WMAP and PLANCK observe consistent large-angle CMB temperature patterns, however, restricts the possibilities for anomalies of purely instrumental origin to features shared by the two experiments. This still leaves room, say, for calibration issues that insist on the CMB dipole.

These investigations were inspired by pre-inflationary scenarios that occur in String Theory, where the inflaton decelerates to slow-roll after bouncing against a steep exponential potential. The transition to slow-roll introduces a universal $k^{3}$ - cut at low frequencies in primordial power spectra, which is captured by eq. (23). The cut is generally accompanied by a narrow peak and a few oscillations, whose detailed features are model dependent. In [16 we found at most a scant evidence for a peak located at the transition: these local effects, if they are there, appear currently beyond reach. Still, the oscillations would 
be a natural origin of the even-odd asymmetry. At any rate, relics of the approach to slow-roll would open up an enticing window on the earliest stages of inflation, when sizable primordial inhomogeneities were possibly around. Or, perhaps less enticingly, the power depression might be revealing local features of the potential that were experienced by the inflaton at a later epoch.

Pre-inflationary scenarios involving a decelerating inflaton, and in particular the string-inspired ones of [23]-26], would have another signature that is potentially interesting: larger values of the tensor-to-scalar ratio $r$ should present themselves in the low- $\ell$ region $(\ell \lesssim 20$, according to the typical values of $\Delta$ identified in [16] or here), where however the amplitudes of both scalar and tensor perturbations would be somewhat reduced. For instance, in [24] we showed that when an inflaton approaches slow-roll in an exponential potential, $r$ is larger than its limiting value, and we found numerically a similar behavior in other models. These include a canonically normalized inflaton field in Starobinsky potentials [18, with also an exponential wall contribution that is "critical" in the sense of [23. An example where the inflaton experiences a mild bounce on a "critical" exponential [24] is displayed in fig. 19 Even ignoring the peak in the scalar spectrum, which is not modelled by eq. (23), here $r$ can grow by a factor 5 within a decade that, with a short inflation, could correspond to the first few multipoles, while accounting somehow for a peak structure the growth would enhance to a factor of about 7. Moreover, larger values of $r$ at low $\ell$ are favoured if the deceleration occurs in steeper regions of the potential. All in all, an enhancement of $r$ by about one order of magnitude in the low-multipole region $\ell \lesssim 20$ appears conceivable for this type of dynamics: it would allow tensor-to-scalar ratios $r \sim 3 \times 10^{-2}$ at large scales even in the Starobinsky-like scenarios that are favoured by PLANCK data. Alternatively, an enhancement mechanism of this type could help one place tighter constraints on inflationary scenarios than what was achieved so far by PLANCK.

The enhancement of $r$ is potentially interesting, together with the corresponding high tilt at low $\ell$, as in fig. 19. Regardless of any specific scenario, B-mode detection is a long-awaited result, because it would determine the in- 
flationary scale, but above and beyond all this, because it would also provide some clear, if indirect, evidence for Quantum Gravity. Its effects on interactions are extremely feeble at accessible energies, but its zero-point fluctuations, classicalized by inflation according to [17, ought to be imprinted on the CMB sky. All in all, a properly enhanced $r$ for values of $k \sim \Delta \sim 0.35 \times 10^{-3} \mathrm{Mpc}^{-1}$ might bring $\mathrm{B}-$ mode detection within the reach of planned sub-orbital probes aiming at the CMB re-ionization bump, including the Italian LSPE [45] and the CLASS [46] telescope array, and of the aforementioned satellites LiteBIRD [4] and CORE [47]. In this respect, we have also shown how the high-quality low- $\ell$ polarization data expected from these future surveys may help to improve the determination of $\Delta$ up to the $6 \sigma$ level.

Independently of its dynamical origin, a primordial power spectrum modified along the lines of eq. 23) would also have important consequences for very large galaxy clusters (see, for instance, [50]), whose distribution ought to drop at distance scales corresponding to $\Delta$. If further studies of systematics of astrophysical or instrumental origin will lend support to our findings, the results of [15] and [16], obtained largely from high-latitude Galactic regions, will deserve more attention.

\section{Conclusions}

Let us conclude with a brief summary of the main points touched upon in the preceding sections, which rest largely on a Bayesian approach:

- the even low- $\ell$ CMB multipoles are largely suppressed with respect to their predicted values in $\Lambda \mathrm{CDM}$, in a way that does not depend sizably on the choice of Galactic masking, and thus on the portion of sky under scrutiny. This is confirmed by an analysis that rests on several estimators defined in Section 3.2 , and reflects itself in detection levels of $\Delta$ via even multipoles alone that are essentially independent of Galactic masking;

- the odd low- $\ell$ CMB multipoles are not suppressed, up to a slight decrease at high Galactic latitudes; 
- the combined behavior of even and odd low- $\ell$ CMB multipoles reflects itself in determinations of the cutoff scale $\Delta$ of eq. 23) that improve at higher latitudes, reaching the $3-\sigma$ level in a blind $+30^{\circ}$ extension of the standard PlanCK mask (the slight improvement with respect to [15, 16] is due to a different treatment of low $-\ell$ polarization data, obtained following [31]);

- String Theory and Supergravity can associate to these results enticing scenarios: $\Delta$ might be a relic of a deceleration that resulted in the onset of the inflationary phase, some glimpses of which could be accessible to us with a small-enough number of $e$-folds. If this were the case, the lowfrequency cut of both scalar and tensor spectra would be accompanied, in the same low- $\ell$ region, by a sharp increase of the tensor-to-scalar ratio $r$, with a high tilt, by almost an order of magnitude. Finally, if $\Delta$ captures a real deviation from an almost scale invariant primordial power spectrum, it should be impinging on structure formation at the largest scales [50], which ought to drop accordingly;

- the available low $-\ell$ polarization data have played a role in our determination of the cutoff scale $\Delta$. The next generation of space-borne experiments aimed at high-quality polarization data has the potential to raise it up to the $6-\sigma$ level (or perhaps to rule it out altogether). A positive result could further our understanding of the inflationary paradigm.

Acknowledgments. We acknowledge the use of computing facilities at NERSC (USA), of the HEALPix package [32, and of the PLANCK Legacy Archive (PLA). This research was supported by ASI through the Grant 2016-24-H.0 (COSMOS) and through the ASI/INAF Agreement I/072/09/0 for the Planck LFI Activity of Phase E2, and by INFN (I.S. GSS- Pi, FlaG, InDark). NK was supported in part by the JSPS KAKENHI, Grant Number 26400253 and in part by Scuola Normale, while AS was supported in part by Scuola Normale. We would like to thank APC-Paris VII, Scuola Normale Superiore, the University 
of Ferrara and IASF-Bologna for their kind hospitality.

\section{References}

\section{References}

[1] P. A. R. Ade et al. [Planck Collaboration], Astron. Astrophys. 594 (2016) A13 [arXiv:1502.01589 [astro-ph.CO]].

[2] G. Hinshaw, A. J. Banday, C. L. Bennett, K. M. Gorski, A. Kogut, C. H. Lineweaver, G. F. Smoot and E. L. Wright, Astrophys. J. 464 (1996) L25 [astro-ph/9601061].

[3] C. Copi, D. Huterer, D. Schwarz and G. Starkman, Phys. Rev. D 75 (2007) 023507 [astro-ph/0605135].

[4] C. J. Copi, D. Huterer, D. J. Schwarz and G. D. Starkman, Mon. Not. Roy. Astron. Soc. 399 (2009) 295 [arXiv:0808.3767 [astro-ph]].

[5] D. Sarkar, D. Huterer, C. J. Copi, G. D. Starkman and D. J. Schwarz, Astropart. Phys. 34 (2011) 591 [arXiv:1004.3784 [astro-ph.CO]].

[6] A. Gruppuso, Mon. Not. Roy. Astron. Soc. 437 (2014) no.3, 2076 [arXiv:1310.2822 [astro-ph.CO]].

[7] P. A. R. Ade et al. [Planck Collaboration], Astron. Astrophys. 594 (2016) A16 [arXiv:1506.07135 [astro-ph.CO]].

[8] D. J. Schwarz, C. J. Copi, D. Huterer and G. D. Starkman, Class. Quant. Grav. 33 (2016) no.18, 184001 [arXiv:1510.07929 [astro-ph.CO]].

[9] C. J. Copi, D. Huterer, D. J. Schwarz and G. D. Starkman, Mon. Not. Roy. Astron. Soc. 451 (2015) no.3, 2978 [arXiv:1310.3831 [astro-ph.CO]].

[10] C. Monteserin, R. B. B. Barreiro, P. Vielva, E. Martinez-Gonzalez, M. P. Hobson and A. N. Lasenby, Mon. Not. Roy. Astron. Soc. 387 (2008) 209 [arXiv:0706.4289 [astro-ph]]. 
[11] M. Cruz, P. Vielva, E. Martinez-Gonzalez and R. B. Barreiro, Mon. Not. Roy. Astron. Soc. 412 (2011) 2383 [arXiv:1005.1264 [astro-ph.CO]].

[12] A. Gruppuso, P. Natoli, F. Paci, F. Finelli, D. Molinari, A. De Rosa and N. Mandolesi, JCAP 1307 (2013) 047 [arXiv:1304.5493 [astro-ph.CO]].

[13] C. J. Copi, M. O’Dwyer and G. D. Starkman, Mon. Not. Roy. Astron. Soc. 463 (2016) 3305 arXiv:1605.09732 [astro-ph.CO].

[14] P. A. R. Ade et al. [Planck Collaboration], Astron. Astrophys. 571 (2014) A19 [arXiv:1303.5079 [astro-ph.CO]], Astron. Astrophys. 594 (2016) A21 [arXiv:1502.01595 [astro-ph.CO]].

[15] A. Gruppuso and A. Sagnotti, Int. J. Mod. Phys. D 24 (2015) no.12, 1544008 [arXiv:1506.08093 [astro-ph.CO]].

[16] A. Gruppuso, N. Kitazawa, N. Mandolesi, P. Natoli and A. Sagnotti, Phys. Dark Univ. 11 (2016) 68 [arXiv:1508.00411 [astro-ph.CO]].

[17] V. F. Mukhanov and G. V. Chibisov, JETP Lett. 33 (1981) 532 [Pisma Zh. Eksp. Teor. Fiz. 33 (1981) 549].

[18] A. A. Starobinsky, Phys. Lett. B 91 (1980) 99; D. Kazanas, Astrophys. J. 241 (1980) L59; K. Sato, Phys. Lett. B 99 (1981) 66; A. H. Guth, Phys. Rev. D 23 (1981) 347; A. D. Linde, Phys. Lett. B 108 (1982) 389; A. Albrecht and P. J. Steinhardt, Phys. Rev. Lett. 48 (1982) 1220; A. D. Linde, Phys. Lett. B 129 (1983) 177; For reviews see: N. Bartolo, E. Komatsu, S. Matarrese and A. Riotto, Phys. Rept. 402 (2004) 103 [astro-ph/0406398]; V. Mukhanov, "Physical foundations of cosmology," Cambridge, UK: Univ. Pr. (2005); S. Weinberg, "Cosmology," Oxford, UK: Oxford Univ. Pr. (2008); D. H. Lyth and A. R. Liddle, "The primordial density perturbation: Cosmology, inflation and the origin of structure," Cambridge, UK: Cambridge Univ. Pr. (2009); D. S. Gorbunov and V. A. Rubakov, "Introduction to the theory of the early universe: Cosmological perturbations and inflationary theory," Hackensack, USA: World 
Scientific (2011); J. Martin, C. Ringeval and V. Vennin, Phys. Dark Univ. 5-6 (2014) 75 [arXiv:1303.3787 [astro-ph.CO]].

[19] For reviews see: M. B. Green, J. H. Schwarz and E. Witten, "Superstring Theory", 2 vols., Cambridge, UK: Cambridge Univ. Press (1987); J. Polchinski, "String theory", 2 vols. Cambridge, UK: Cambridge Univ. Press (1998); C. V. Johnson, "D-branes," USA: Cambridge Univ. Press (2003); B. Zwiebach, "A first course in string theory" Cambridge, UK: Cambridge Univ. Press (2004); K. Becker, M. Becker and J. H. Schwarz, "String theory and M-theory: A modern introduction" Cambridge, UK: Cambridge Univ. Press (2007); E. Kiritsis, "String theory in a nutshell", Princeton, NJ: Princeton Univ. Press (2007).

[20] D. Z. Freedman, P. van Nieuwenhuizen and S. Ferrara, Phys. Rev. D 13 (1976) 3214; S. Deser and B. Zumino, Phys. Lett. B 62 (1976) 335.

For a comprehensive review see: D. Z. Freedman and A. Van Proeyen, Cambridge, UK: Cambridge Univ. Pr. (2012) 607 p. A recent elementary review is: S. Ferrara and A. Sagnotti, Riv. Nuovo Cim. 40 (2017) no.6, 1 [J. Phys. Conf. Ser. 873 (2017) no.1, 012014] [arXiv:1702.00743 [hep-th]].

[21] A. Sagnotti, in Cargese '87, "Non-Perturbative Quantum Field Theory", eds. G. Mack et al (Pergamon Press, 1988), p. 521, arXiv:hep-th/0208020; G. Pradisi and A. Sagnotti, Phys. Lett. B 216 (1989) 59; P. Horava, Nucl. Phys. B 327 (1989) 461, Phys. Lett. B 231 (1989) 251; M. Bianchi and A. Sagnotti, Phys. Lett. B 247 (1990) 517, Nucl. Phys. B 361 (1991) 519; M. Bianchi, G. Pradisi and A. Sagnotti, Nucl. Phys. B 376 (1992) 365; A. Sagnotti, Phys. Lett. B 294 (1992) 196 [arXiv:hep-th/9210127]. For reviews see: E. Dudas, Class. Quant. Grav. 17 (2000) R41 [arXiv:hepph/0006190]; C. Angelantonj and A. Sagnotti, Phys. Rept. 371 (2002) 1 [Erratum-ibid. 376 (2003) 339] [arXiv:hep-th/0204089].

[22] S. Sugimoto, Prog. Theor. Phys. 102 (1999) 685 [arXiv:hep-th/9905159]; I. Antoniadis, E. Dudas and A. Sagnotti, Phys. Lett. B 464 (1999) 
38 [arXiv:hep-th/9908023]; C. Angelantonj, Nucl. Phys. B 566 (2000) 126 [arXiv:hep-th/9908064]; G. Aldazabal and A. M. Uranga, JHEP 9910 (1999) 024 [arXiv:hep-th/9908072]; C. Angelantonj, I. Antoniadis, G. D'Appollonio, E. Dudas and A. Sagnotti, Nucl. Phys. B 572 (2000) 36 [arXiv:hep-th/9911081].

[23] E. Dudas, N. Kitazawa and A. Sagnotti, Phys. Lett. B 694 (2010) 80 [arXiv:1009.0874 [hep-th]]; A. Sagnotti, Phys. Part. Nucl. Lett. 11 (2014) 836 [arXiv:1303.6685 [hep-th]]; P. Fré, A. Sagnotti and A. S. Sorin, Nucl. Phys. B 877 (2013) 1028 [arXiv:1307.1910 [hep-th]].

[24] E. Dudas, N. Kitazawa, S. P. Patil and A. Sagnotti, JCAP 1205 (2012) 012 [arXiv:1202.6630 [hep-th]]; N. Kitazawa and A. Sagnotti, JCAP 1404 (2014) 017 [arXiv:1402.1418 [hep-th]], EPJ Web Conf. 95 (2015) 03031 [arXiv:1411.6396 [hep-th]], Mod. Phys. Lett. A 30 (2015) no.28, 1550137 [arXiv:1503.04483 [hep-th]].

[25] A. D. Linde, Phys. Rev. D 59 (1999) 023503 [hep-ph/9807493]; C. R. Contaldi, M. Peloso, L. Kofman and A. D. Linde, JCAP 0307 (2003) 002 [astro-ph/0303636]; Y. -S. Piao, B. Feng and X. -m. Zhang, Phys. Rev. D 69 (2004) 103520 [hep-th/0310206]; Y. -S. Piao, Phys. Rev. D 71 (2005) 087301 [astro-ph/0502343]; D. Boyanovsky, H. J. de Vega and N. G. Sanchez, Phys. Rev. D 74 (2006) 123007 [astro-ph/0607487]; C. Destri, H. J. de Vega and N. G. Sanchez, Phys. Rev. D 78 (2008) 023013 [arXiv:0804.2387 [astro-ph]]; F. J. Cao, H. J. de Vega and N. G. Sanchez, Phys. Rev. D 78 (2008) 083508 [arXiv:0809.0623 [astro-ph]]; R. K. Jain, P. Chingangbam, J. -O. Gong, L. Sriramkumar and T. Souradeep, JCAP 0901 (2009) 009 [arXiv:0809.3915 [astro-ph]]; E. Ramirez and D. J. Schwarz, Phys. Rev. D 80 (2009) 023525 [arXiv:0903.3543 [astroph.CO]], Phys. Rev. D 85 (2012) 103516 [arXiv:1111.7131 [astro-ph.CO]]; R. K. Jain, P. Chingangbam, L. Sriramkumar and T. Souradeep, Phys. Rev. D 82 (2010) 023509 [arXiv:0904.2518 [astro-ph.CO]]; C. Destri, H. J. de Vega and N. G. Sanchez, Phys. Rev. D 81 (2010) 063520 
[arXiv:0912.2994 [astro-ph.CO]]; E. Ramirez, Phys. Rev. D 85 (2012) 103517 [arXiv:1202.0698 [astro-ph.CO]]; Z.-G. Liu, Z.-K. Guo and Y.S. Piao, Phys. Rev. D 88 (2013) 063539 [arXiv:1304.6527 [astroph.CO]]; M. Cicoli, S. Downes and B. Dutta, JCAP 1312 (2013) 007 [arXiv:1309.3412 [hep-th], arXiv:1309.3412]; F. G. Pedro and A. Westphal, JHEP 1404 (2014) 034 [arXiv:1309.3413 [hep-th]]; R. Bousso, D. Harlow and L. Senatore, Phys. Rev. D 91 (2015) no.8, 083527 [arXiv:1309.4060 [hep-th]]; M. Cicoli, S. Downes, B. Dutta, F. G. Pedro and A. Westphal, JCAP 1412 (2014) no.12, 030 [arXiv:1407.1048 [hepth]]; Z. G. Liu, Z. K. Guo and Y. S. Piao, Eur. Phys. J. C 74 (2014) no.8, 3006 [arXiv:1311.1599 [astro-ph.CO]]; A. Y. Kamenshchik, A. Tronconi and G. Venturi, JCAP 1504 (2015) 04, 046 [arXiv:1501.06404 [gr-qc]]; Y. F. Cai, E. G. M. Ferreira, B. Hu and J. Quintin, Phys. Rev. D 92 (2015) no.12, 121303 [arXiv:1507.05619 [astro-ph.CO]]; Q. Yang, Y. Liu and H. Di, Phys. Rev. D 96, no. 8, 083516 (2017) [arXiv:1612.03708 [astro-ph.CO]].

[26] A. Sagnotti, Mod. Phys. Lett. A 32 (2016) no.01, 1730001 [Subnucl. Ser. 53 (2017) 289] [arXiv:1509.08204 [astro-ph.CO]]; J. Mourad and A. Sagnotti, arXiv:1711.11494 [hep-th].

[27] N. Aghanim et al. [Planck Collaboration], Astron. Astrophys. 594 (2016) A11 [arXiv:1507.02704 [astro-ph.CO]].

[28] H. K. Eriksen et al., Astrophys. J. Suppl. 155 (2004) 227 [astro$\mathrm{ph} / 0407028]$.

[29] H. K. Eriksen, J. B. Jewell, C. Dickinson, A. J. Banday, K. M. Gorski and C. R. Lawrence, Astrophys. J. 676 (2008) 10 [arXiv:0709.1058].

[30] R. Adam et al. [Planck Collaboration], Astron. Astrophys. 594 (2016) A9 [arXiv:1502.05956 [astro-ph.CO]].

[31] M. Lattanzi et al., JCAP 1702, no. 02, 041 (2017) [arXiv:1611.01123 [astroph.CO]]. 
[32] K. M. Gorski, E. Hivon, A. J. Banday, B. D. Wandelt, F. K. Hansen, M. Reinecke and M. Bartelman, Astrophys. J. 622 (2005) 759 [astro-ph/0409513], http://sourceforge.net/projects/healpix/.

[33] D. N. Spergel et al. [WMAP Collaboration], Astrophys. J. Suppl. 148, 175 (2003) [astro-ph/0302209].

[34] C. J. Copi, D. Huterer, D. J. Schwarz and G. D. Starkman, Mon. Not. Roy. Astron. Soc. 418, 505 (2011) [arXiv:1103.3505 [astro-ph.CO]].

[35] J. Kim and P. Naselsky, Astrophys. J. 714 (2010) L265 [arXiv:1001.4613 [astro-ph.CO]].

[36] J. Kim and P. Naselsky, Phys. Rev. D 82 (2010) 063002 [arXiv:1002.0148 [astro-ph.CO]].

[37] A. Gruppuso, F. Finelli, P. Natoli, F. Paci, P. Cabella, A. De Rosa and N. Mandolesi, Mon. Not. Roy. Astron. Soc. 411 (2011) 1445 [arXiv:1006.1979 [astro-ph.CO]].

[38] P. K. Aluri and P. Jain, Mon. Not. Roy. Astron. Soc. 419 (2012) 3378 [arXiv:1108.5894 [astro-ph.CO]]; P. K. Aluri, J. P. Ralston and A. Weltman, Mon. Not. Roy. Astron. Soc. 472 (2017) no.2, 2410 [arXiv:1703.07070 [astro-ph.CO]].

[39] M. Tegmark and A. de Oliveira-Costa, Phys. Rev. D 64 (2001) 063001 doi:10.1103/PhysRevD.64.063001 [astro-ph/0012120].

[40] A. Lewis and S. Bridle, Phys. Rev. D 66 (2002) 103511 [astro-ph/0205436].

[41] A. Lewis, A. Challinor and A. Lasenby, Astrophys. J. 538 (2000) 473 [astroph/9911177].

[42] A. Gruppuso, A. De Rosa, P. Cabella, F. Paci, F. Finelli, P. Natoli, G. de Gasperis and N. Mandolesi, Mon. Not. Roy. Astron. Soc. 400 (2009) 463 [arXiv:0904.0789 [astro-ph.CO]]. 
[43] N. Aghanim et al. [Planck Collaboration], Astron. Astrophys. 596 (2016) A107 [arXiv:1605.02985 [astro-ph.CO]].

[44] T. Matsumura et al., J. Low. Temp. Phys. 176 (2014) 733 [arXiv:1311.2847 [astro-ph.IM]].

[45] S. Aiola et al. [LSPE Collaboration], Proc. SPIE Int. Soc. Opt. Eng. 8446 (2012) 84467A [arXiv:1208.0281 [astro-ph.IM]].

[46] T. Essinger-Hileman et al., Proc. SPIE Int. Soc. Opt. Eng. 9153, 91531I (2014) [arXiv:1408.4788 [astro-ph.IM]].

[47] J. Delabrouille et al. [CORE Collaboration], arXiv:1706.04516 [astroph.IM].

[48] M. Remazeilles et al. [CORE Collaboration], arXiv:1704.04501 [astroph.CO].

[49] P. Natoli et al. [CORE Collaboration], arXiv:1707.04224 [astro-ph.CO].

[50] M. Ballardini, F. Finelli, C. Fedeli and L. Moscardini, JCAP 1610 (2016) 041 [arXiv:1606.03747 [astro-ph.CO]]. 
standard mask

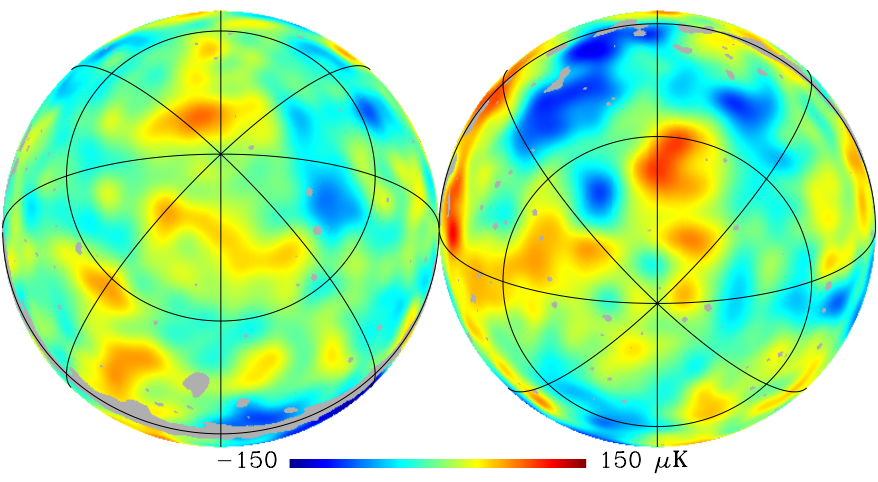

extended mask

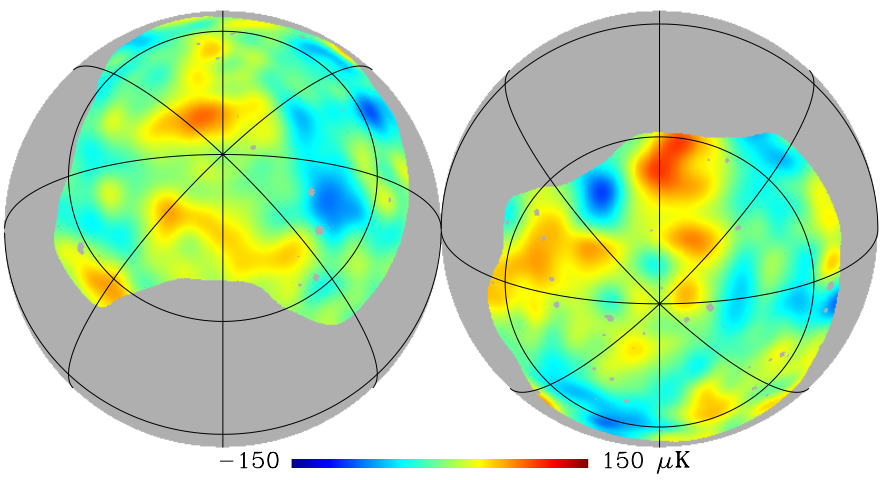

complementary mask

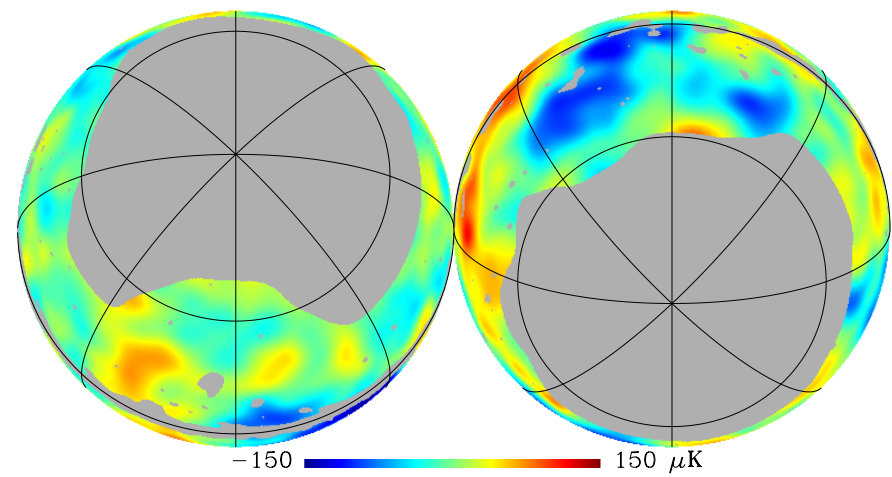

Figure 1: The PLANCK CMB temperature map provided by the Commander algorithm, with three different maskings. From upper to lower panel: standard mask (case $a$ ), extended mask (case $f$ ), complementary mask (case $j$ ). The corresponding observed sky fractions are $93.6 \%, 39.4 \%$ and $54.2 \%$ respectively. Color code units: $\mu K$. 

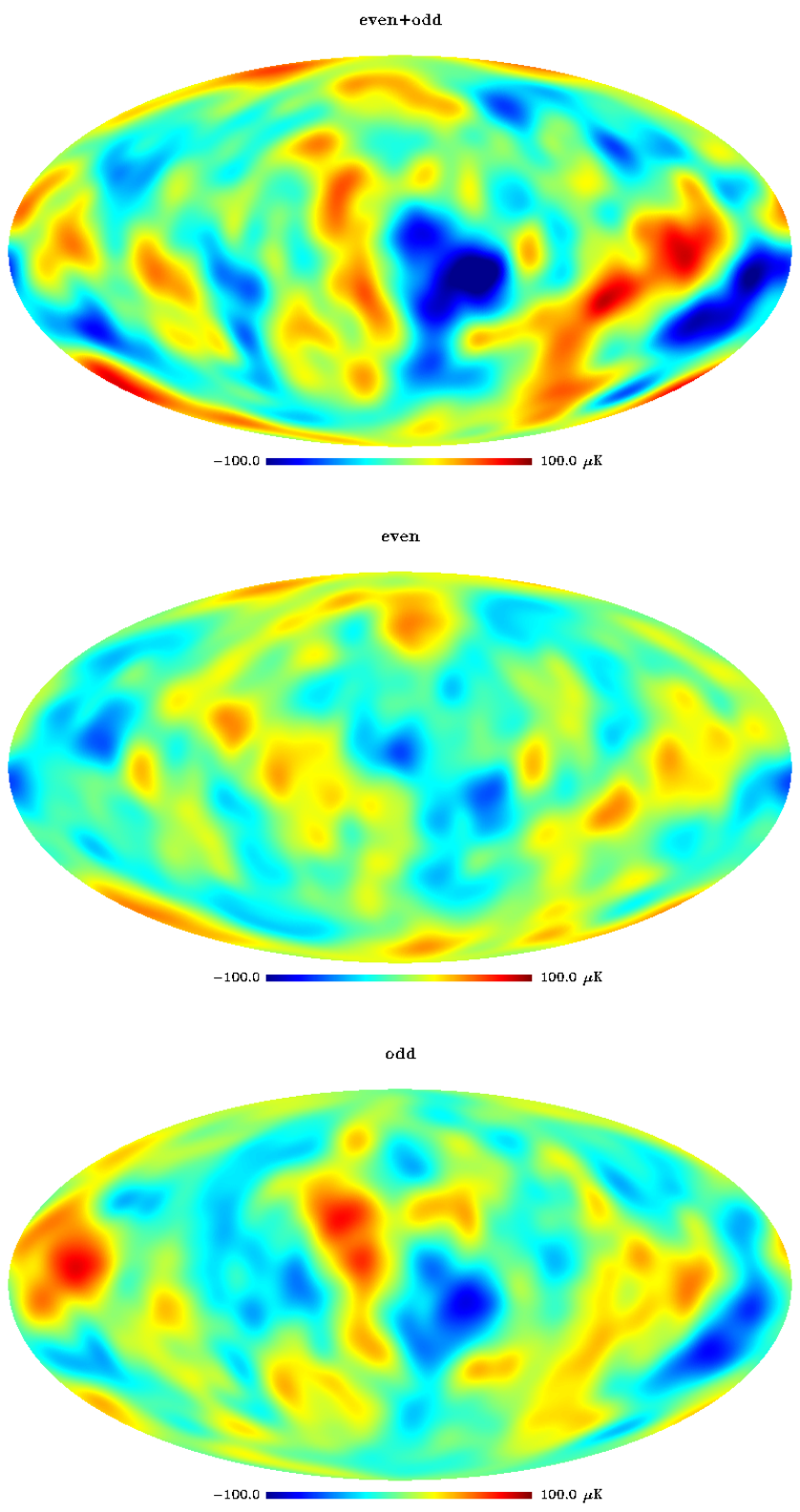

Figure 2: The PlANCK CMB temperature map provided by the Commander algorithm, smoothed at $440^{\prime}$ and reconstructed at $N_{\text {side }}=16$. Upper panel: the full map m; middle panel: its even-parity projection $\mathbf{m}_{+}$; lower panel: its odd-parity projection $\mathbf{m}_{-}$. Color code units: $\mu K$. The reader will not fail to spot eye-catching differences between the symmetrized low- $\ell$ map in the middle panel and the others. 


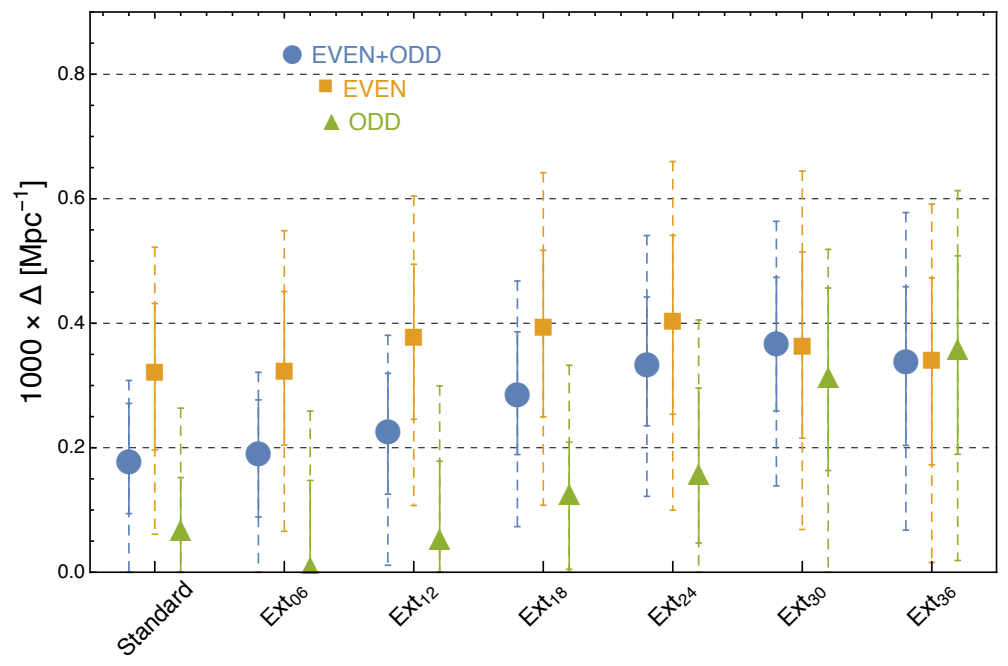

Figure 3: Constraints on $\Delta$ for the standard and extended masks. Estimates and error bars are shown in blue ("even + odd") when all the low- $\ell$ temperature information is taken into account and in orange (green) when only the even (odd) multipoles are considered. In all cases, it should be understood that the low- $\ell$ information is complemented by the high- $\ell$ PLANCK TT likelihood. Solid (dashed) error bars stand for $68 \%(95 \%)$ Bayesian credible intervals. Notice the slight change of pattern for the "odd" determinations around $\mathrm{Ext}_{06}$, which will reflect itself on the behavior of the even-odd asymmetry in fig. 5. 


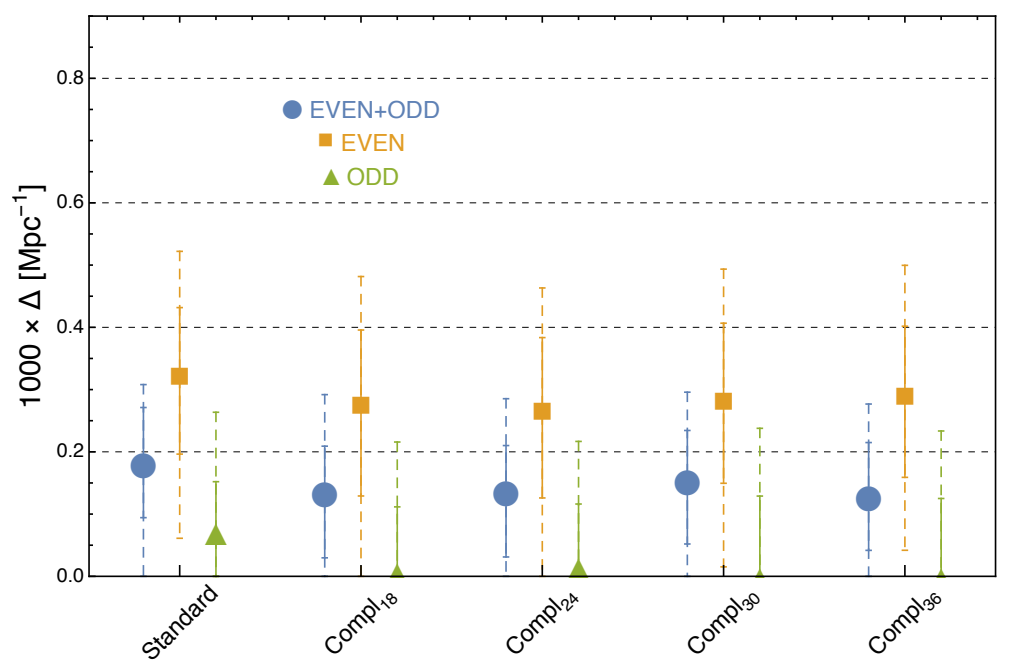

Figure 4: Same as fig. 3, for the standard and complementary masks.

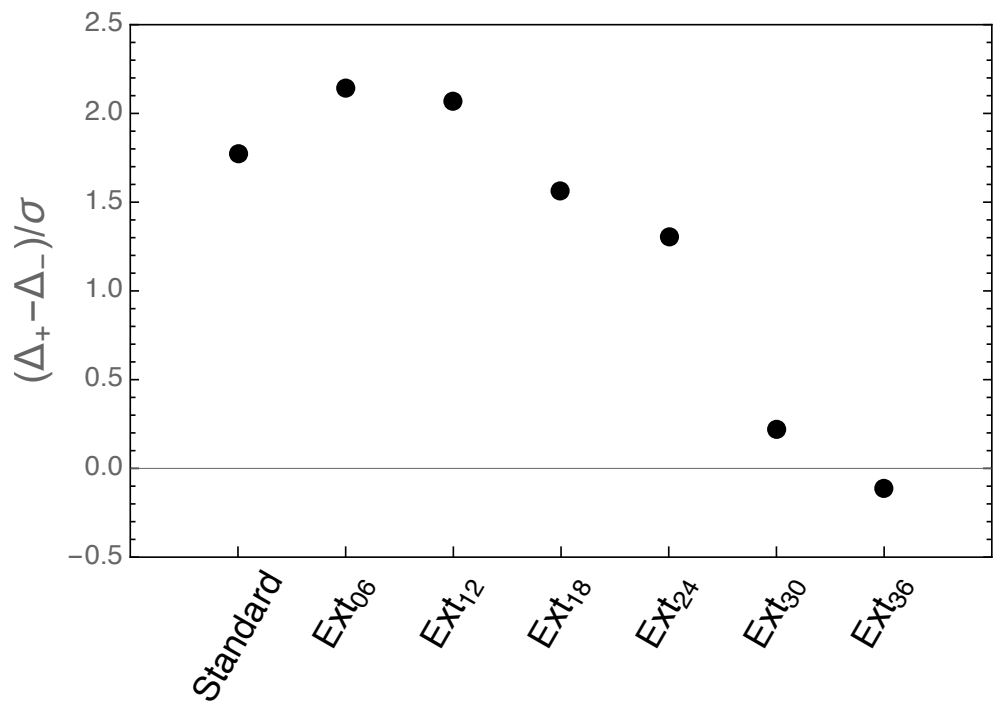

Figure 5: Even-odd asymmetry traced by the cut-off scale $\Delta$, according to eq. 21. 

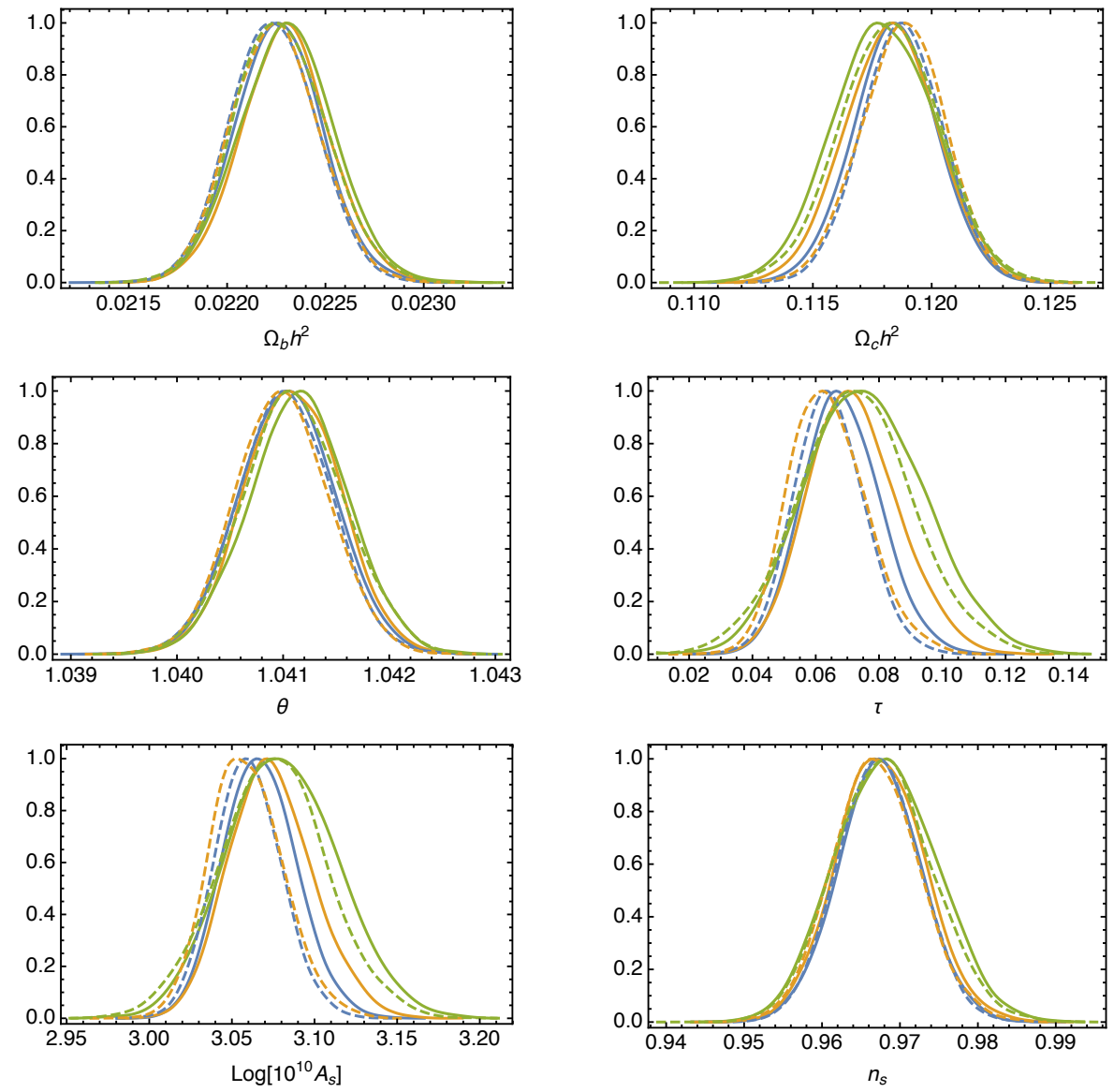

Figure 6: Posterior distribution functions for the $\Lambda$ CDM parameters considering the standard mask. As before, blue, orange and green curves refer to even+odd, even and odd contribution for the low- $\ell$ likelihood. Solid curves refer to the $\Lambda$ CDM $\Delta$ model, while dashed ones refer to the $\Lambda$ CDM model. 

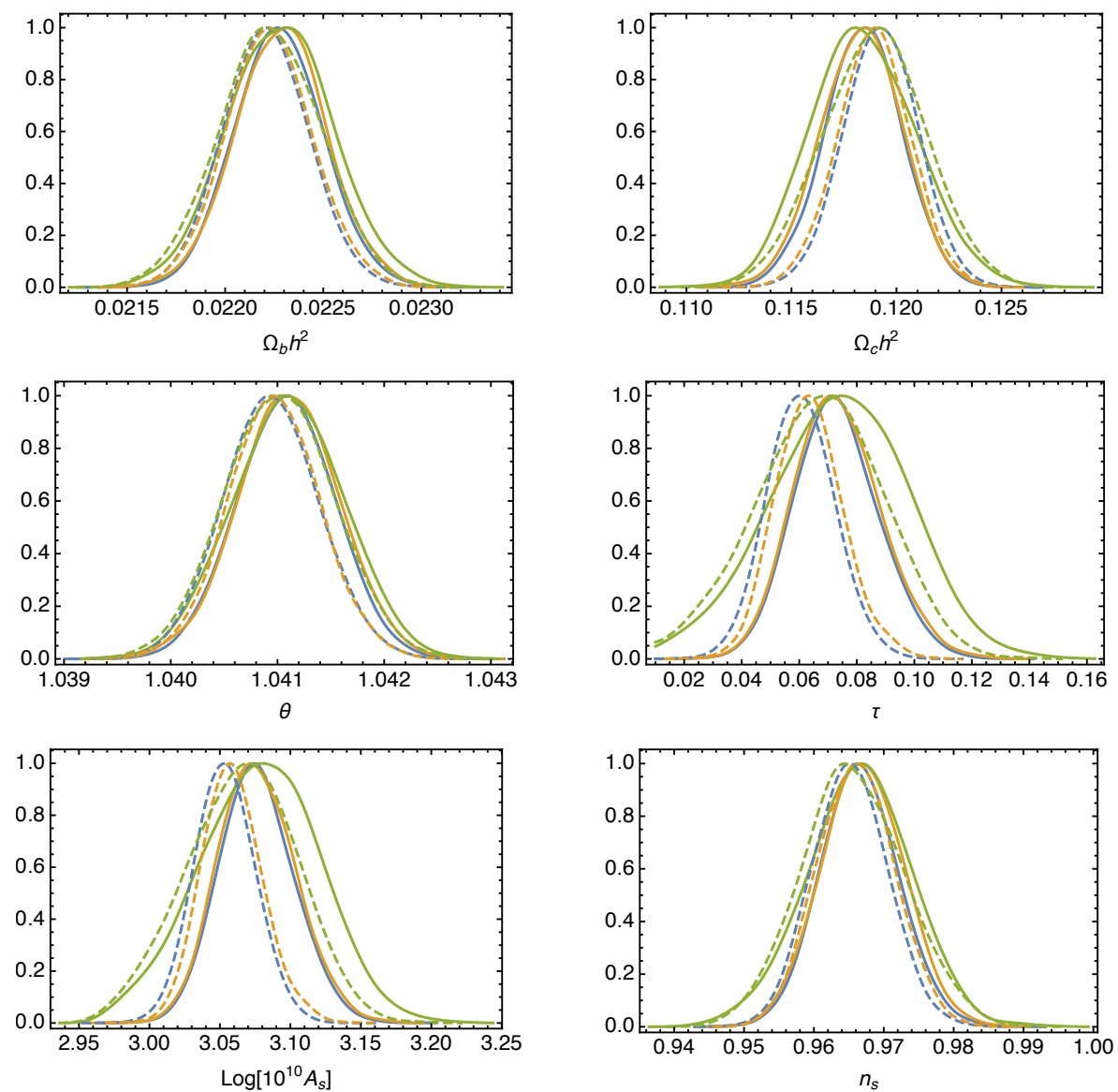

Figure 7: Posterior distribution functions for the $\Lambda$ CDM parameters considering the Ext $_{30}$ mask. Same conventions as in fig. 6 


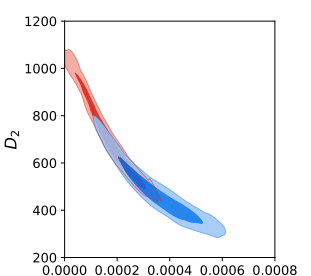

$\Delta$

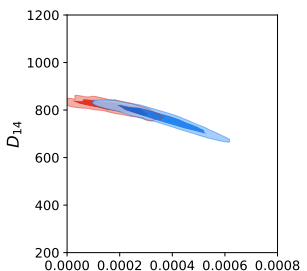

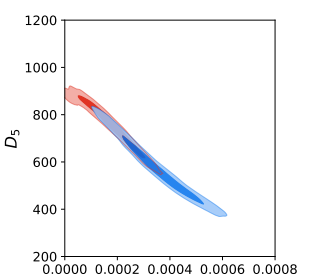

$\Delta$

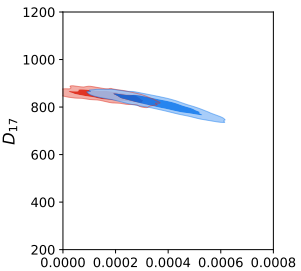

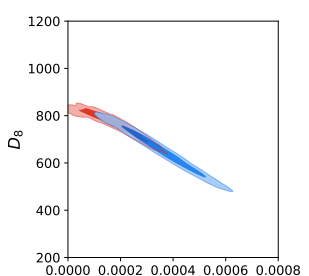

$\Delta$

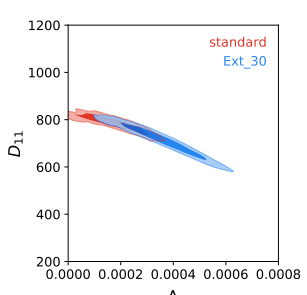

Figure 8: Two-dimensional contour plots for $\Delta$ vs $D_{\ell}=\frac{\ell(\ell+1)}{2 \pi} C_{\ell}$, for $\ell=$ $2,5,8,11,14,17$ and 20 . The red contours are for the standard mask, while the blue ones are for the Ext30 mask.
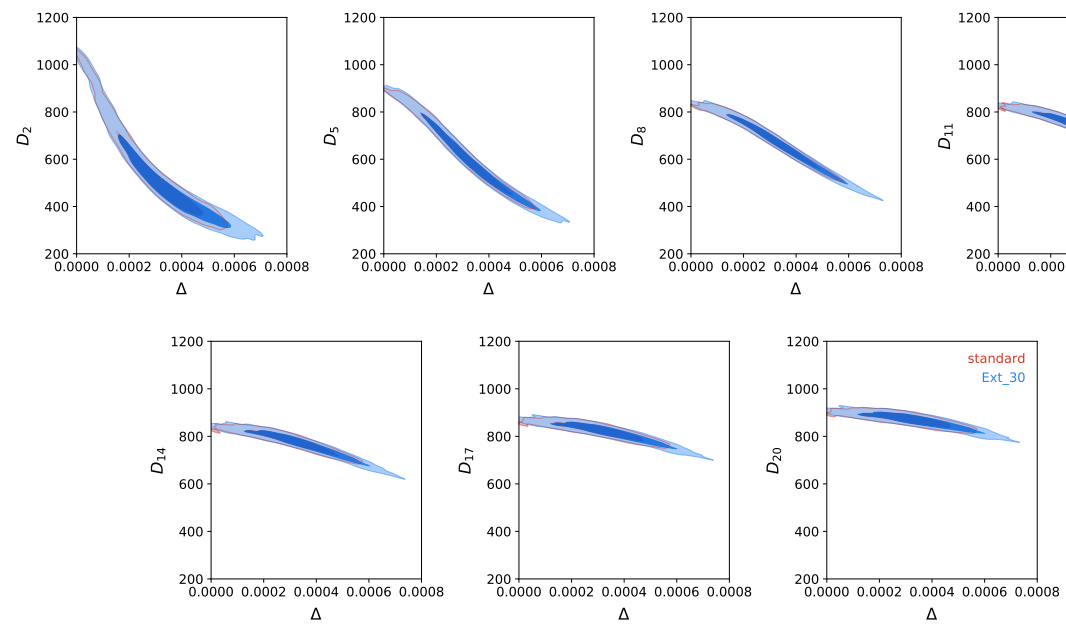

Figure 9: Two-dimensional contour plots for $\Delta$ vs $D_{\ell}=\frac{\ell(\ell+1)}{2 \pi} C_{\ell}$, for $\ell=$ $2,5,8,11,14,17$ and 20, obtained with the "even" part of the likelihood function. The barely visible red contours, which are mostly covered by the larger blue ones, are for the standard mask, while the blue contours are for the $\operatorname{Ext}_{30}$ mask. 

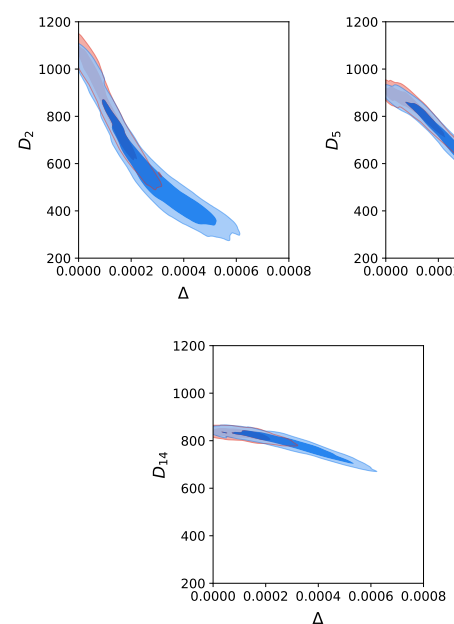
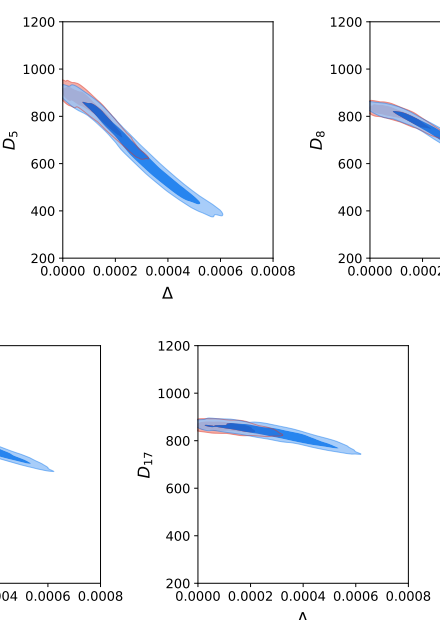
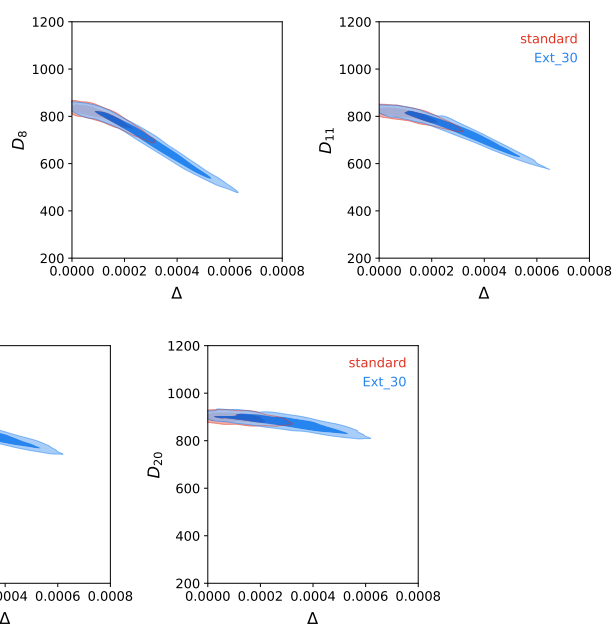

Figure 10: Two-dimensional contour plots for $\Delta$ vs $D_{\ell}=\frac{\ell(\ell+1)}{2 \pi} C_{\ell}$, for $\ell=$ $2,5,8,11,14,17$ and 20, obtained with the "odd" part of the likelihood function. The barely visible red contours, which are mostly covered by the larger blue ones, are for the standard mask, while the blue contours are for the Ext30 mask. 

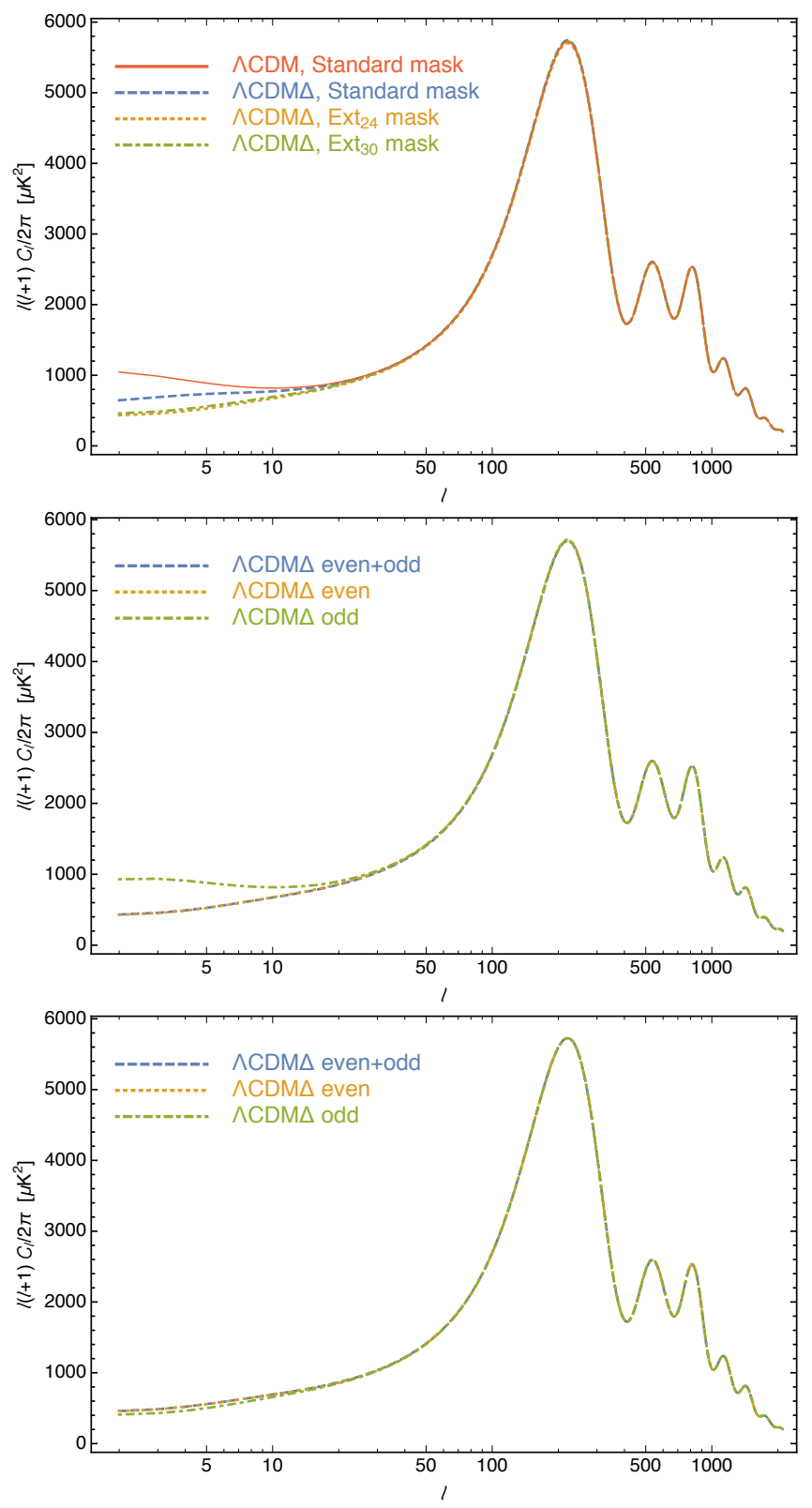

Figure 11: Fiducial models obtained with even+odd $\ell<30$ datasets corresponding to the Standard mask, to the $\operatorname{Ext}_{24}$ mask and to the $\operatorname{Ext}_{30}$ mask (upper panel). Comparison of the fiducial models obtained with even+odd, even and odd $\ell<30$ datasets for the $\operatorname{Ext}_{24}$ mask (middle panel), and for the Ext 30 mask (lower panel). 


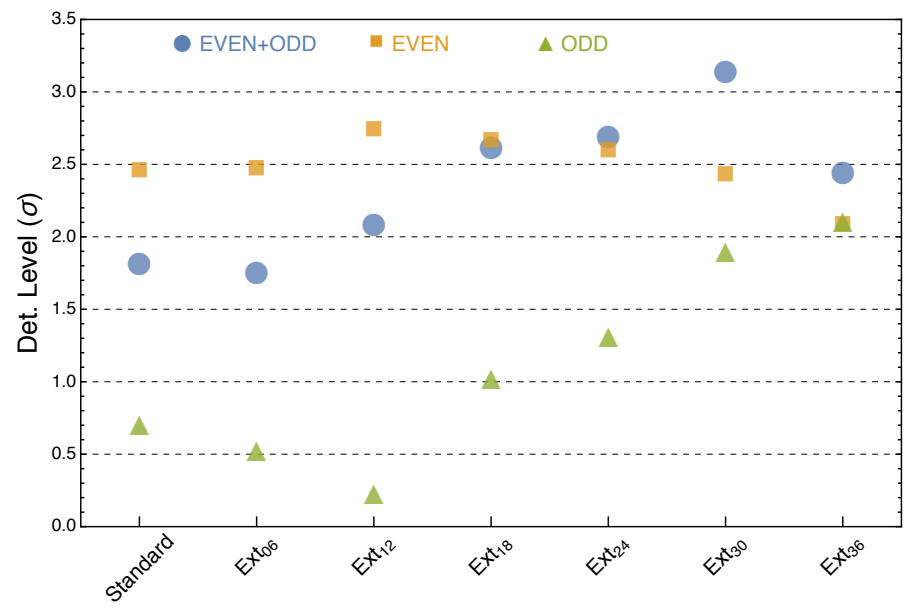

Figure 12: Detection levels of $\Delta$ in units of $\sigma$ for the full dataset (blue circles), even dataset (orange squares) and odd dataset (green triangles), for the various masks of Table. 5
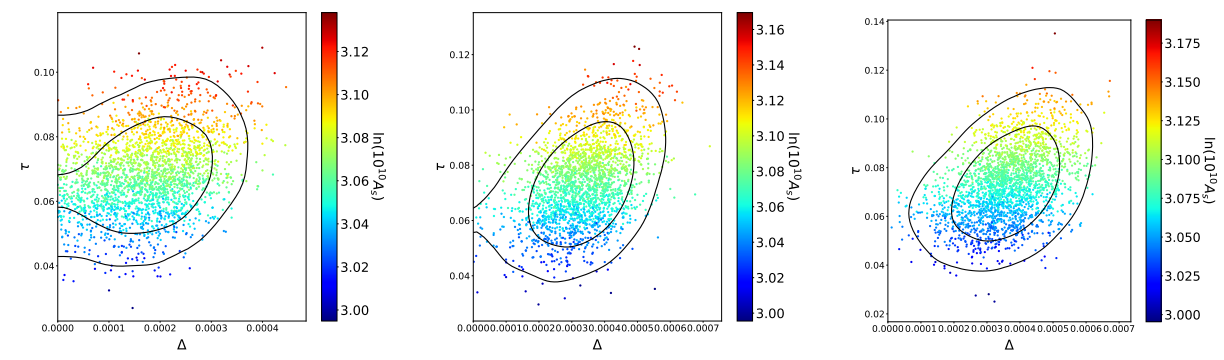

Figure 13: Correlations between $\tau, \Delta$ and $A_{s}$ in the standard mask (left panel), in the $\operatorname{Ext}_{24}$ mask (middle panel) and in the $\operatorname{Ext}_{30}$ mask (right panel). 

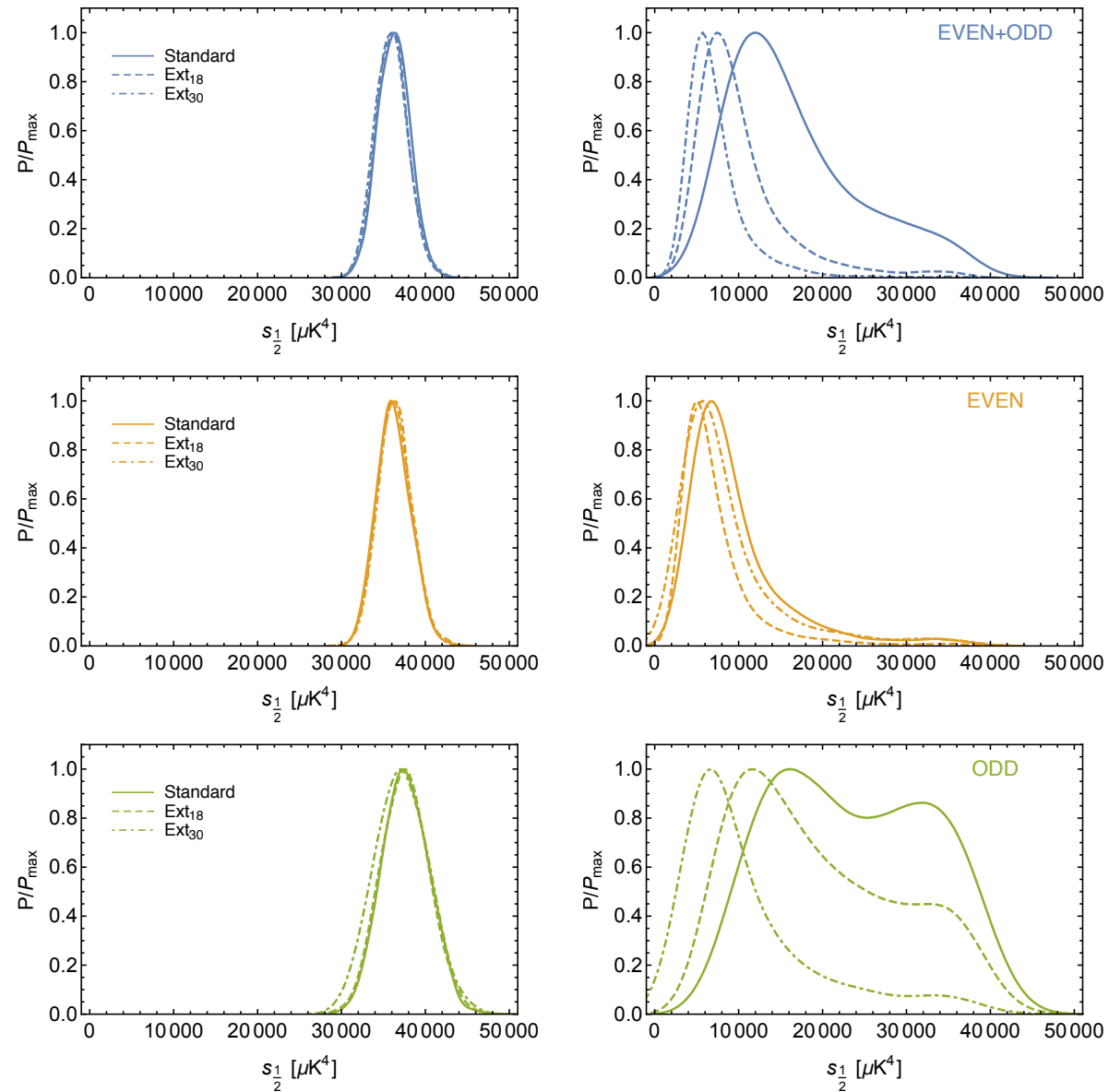

Figure 14: Posterior distribution functions of $S_{\frac{1}{2}}$. The left panels refer to $\Lambda$ CDM and the right ones refer to $\Lambda$ CDM $\Delta$. The upper panels are for "even+odd", the middle ones are for "even" and the lower ones are for "odd". The three types of curves (continuous, dashed and dashed-dotted) are for the Standard mask, the Ext $_{18}$ mask and the Ext 30 mask. 

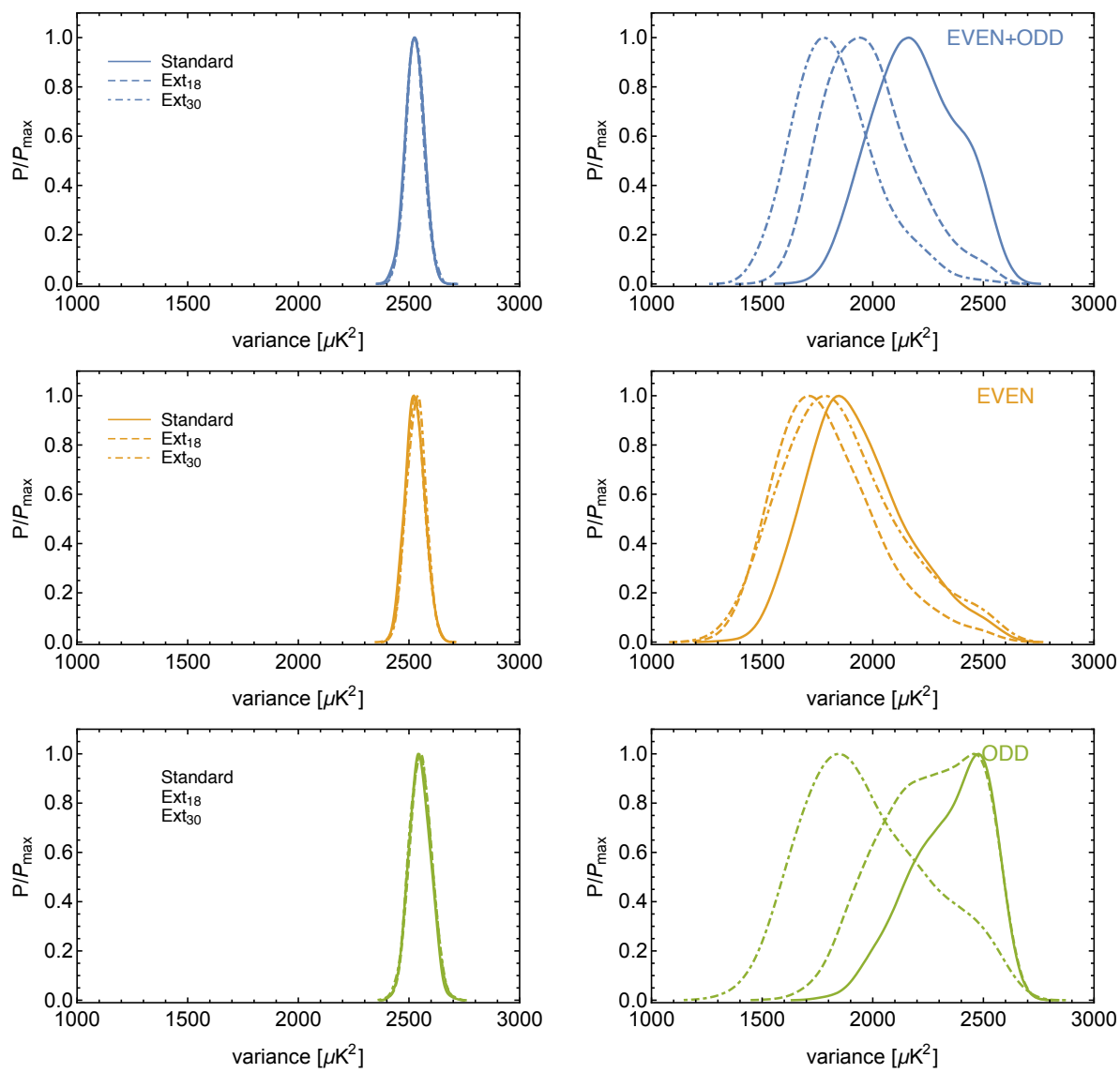

Figure 15: Posterior distribution functions of $V$, with the same conventions as in fig. 14 . 

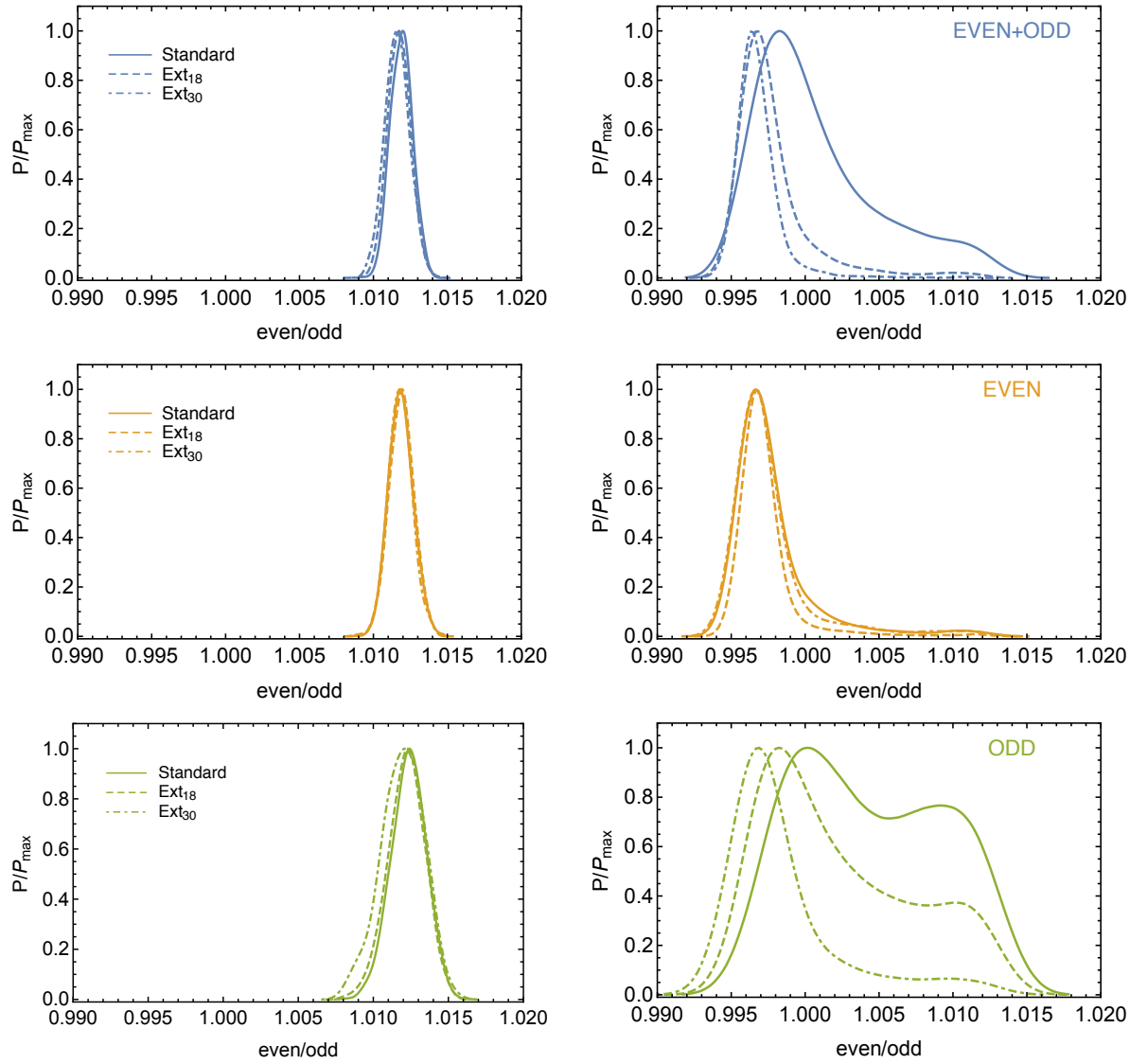

Figure 16: Posterior distribution functions of $R$, with the same conventions as in fig. 14 . 


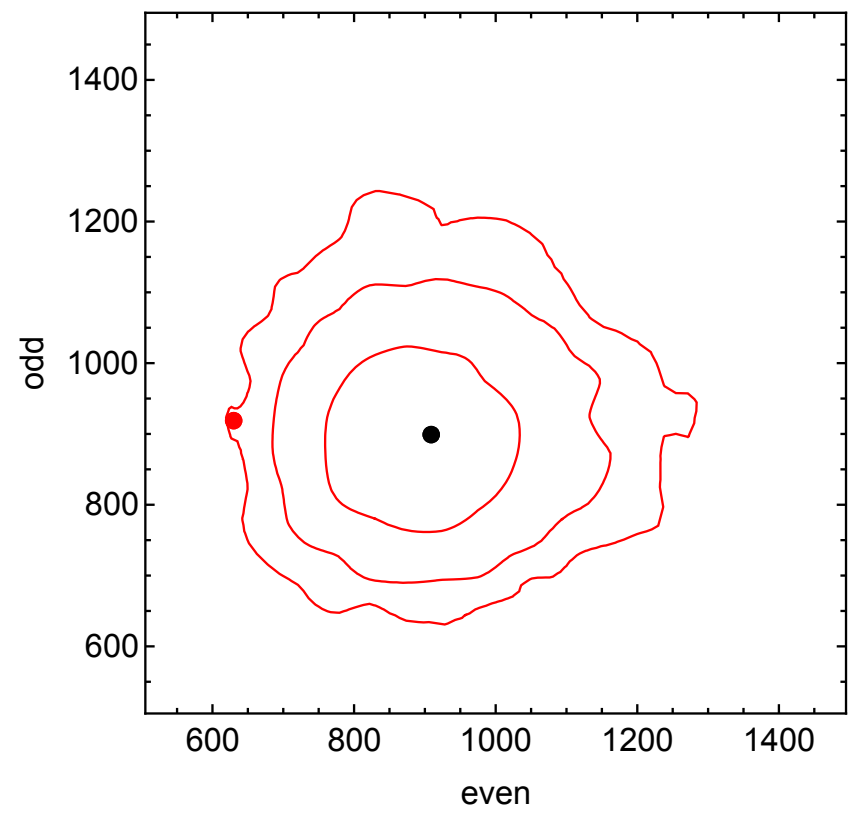

Figure 17: $C^{+}$vs $C^{-}$in a frequentist approach. The black dot identifies the expected values of $C^{+}$and $C^{-}$in $\Lambda$ CDM, while the red one identifies the observed values. The contours elicit the 1,2 and $3 \sigma$ levels expected in $\Lambda \mathrm{CDM}$, in units of $\mu \mathrm{K}^{2}$. 


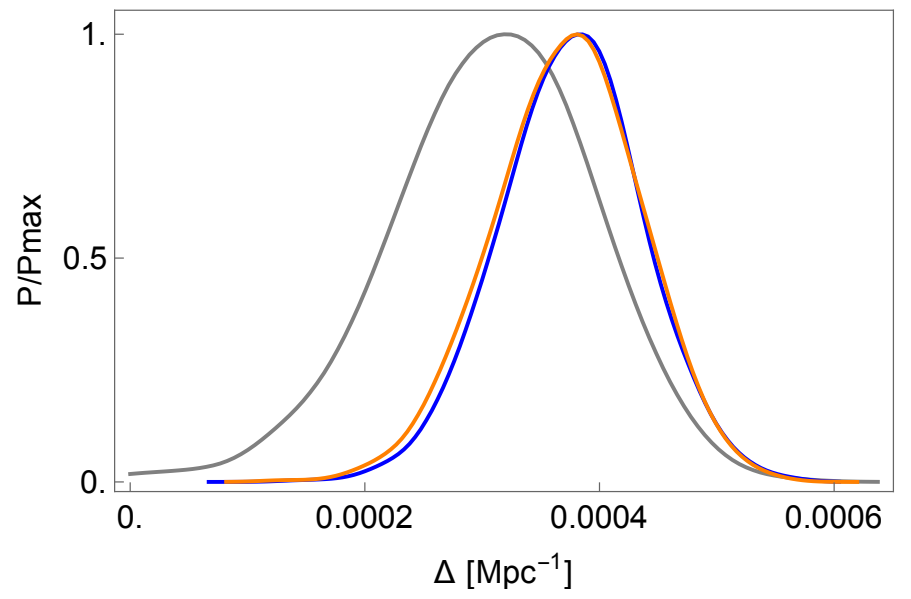

Figure 18: Expected estimates for $\Delta$ from future polarization-oriented experiments. The curves share the same underlying CMB realization, generated from a fiducial cosmological model with $\Delta=0.37 \times 10^{-3}$. With PLANCK-like noise, standard mask in temperature and union mask in polarization, the detection level for $\Delta$ could grow up to about $3.5 \sigma$ (grey curve). With cosmic-variance-limited temperature and polarization data at large scales and the Ext $_{30}$ masking, the detection level for $\Delta$ could rise up to about $6 \sigma$ (orange curve). Finally, with the same cosmic-variance-limited data, the standard mask in temperature and no mask in polarization, the detection level could increase even slightly beyond $6 \sigma$ (blue curve). 

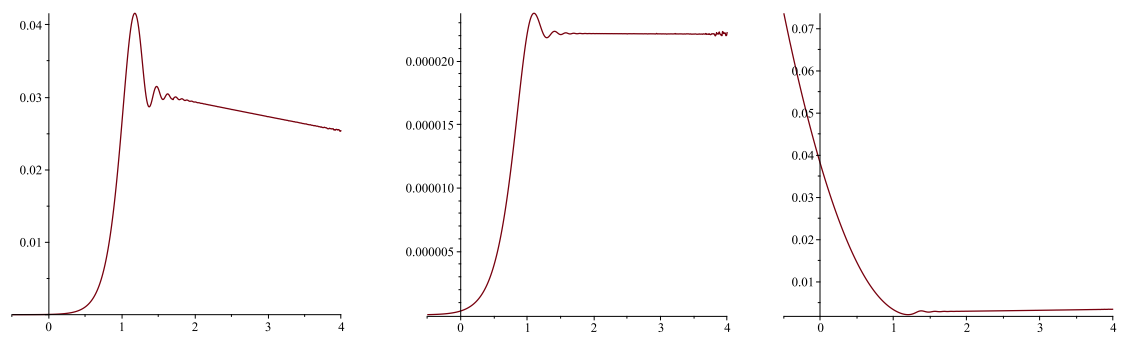

Figure 19: Primordial power spectrum of scalar perturbations (left), of tensor perturbations (center), and corresponding behavior of the tensor-to-scalar ratio $r$ (right) for a Starobinsky model with a "critical" exponential wall, as in 23 . The vertical units are arbitrary, but are the same for tensor and scalar signals. The horizontal unit is $\log \left(k / k_{0}\right)$, with $k_{0}$ corresponding to the scale $\Delta$ of eq $(23)$. In this example $r$ would increase by a factor $5-7$ for $\ell \lesssim 20$. 